\title{
UNA MEDIDA DE INTENSIDAD SÍSMICA QUE PREDICE EL COMPORTAMIENTO NO LINEAL Y EL EFECTO DE LOS MODOS SUPERIORES
}

\author{
Edén Bojórquez Mora ${ }^{(1)}$, Robespierre Chávez López ${ }^{(2)}$, \\ Sonia E. Ruiz Gómez ${ }^{(3)}$ y Alfredo Reyes Salazar ${ }^{(1)}$
}

\begin{abstract}
RESUMEN
El objetivo de este trabajo es analizar la eficiencia de una medida de intensidad sísmica $(I S)$ capaz de predecir tanto el comportamiento no lineal como el efecto de los modos superiores de estructuras sometidas a sismos de diversas características. La medida definida como intensidad de Bojórquez está inspirada en un parámetro para caracterizar la forma espectral denominado $N_{p}$. Aunque normalmente la forma espectral más utiliza es en términos de la pseudo-aceleración, en este trabajo con la finalidad de mejorar la eficiencia de la medida de intensidad, se utilizan otros parámetros tales como velocidad y desplazamiento. La eficiencia de la medida de intensidad sísmica aquí presentada se obtiene al someter varias edificaciones de acero a movimientos sísmicos registrados en suelos con distintas características. Se concluye que el parámetro analizado muestra buena relación con la respuesta estructural y es más eficiente que otras medidas de intensidad sísmica usadas frecuentemente.
\end{abstract}

Palabras clave: intensidad sísmica; forma espectral; eficiencia; respuesta sísmica; marcos de acero

\section{A GROUND MOTION INTENSITY MEASURE TO PREDICT NONLINEAR BEHAVIOR AND HIGHER MODE EFFECTS}

\begin{abstract}
The aim of this paper is to analyze the Bojórquez ground motion intensity measure in order to improve its efficiency to predict both the nonlinear behavior and the higher modes effects of structures subjected to earthquakes of different characteristics. The intensity measure is generally based on a proxy of the spectral shape named $N_{p}$. Although the most commonly spectral shape used is in term of the spectral acceleration, in this paper with the aim to improve the efficiency of the

Artículo recibido el 8 de noviembre de 2012 y aprobado para su publicación el 24 de abril de 2014. Se aceptarán comentarios y/o discusiones hasta cinco meses después de su publicación.

(1) Profesor Facultad de Ingeniería, Universidad Autónoma de Sinaloa, Calzada de las Américas y Boulevard Universitarios S/N, Ciudad Universitaria, Culiacán, Sinaloa, México, C.P. 80040. eden@uas.edu.mx eden_bmseg@hotmail.com $\underline{\text { reyes@uas.edu.mx }}$

(2) Estudiante Facultad de Ingeniería, Universidad Autónoma de Sinaloa, Calzada de las Américas y Boulevard Universitarios S/N, Ciudad Universitaria, Culiacán, Sinaloa, México, C.P. 80040. robespierre chavez@ hotmail.com

(3) Profesor Instituto de Ingeniería, Universidad Nacional Autónoma de México, Coyoacán, C.P. 04510, México, D.F. sruizg@iingen.unam.mx
\end{abstract}


intensity measure, other parameters such as velocity and displacement are selected. The efficiency of the intensity measure here presented is computed by means of several steel buildings subjected to ground motions recorded in soils with different characteristics. It is concluded that the parameter presented here in shows good relation with the structural response of buildings as compared with ground other motion intensity measures commonly used.

Keywords: intensity measure; spectral shape; efficiency; seismic response; steel frames

\section{INTRODUCCIÓN}

La definición de un parámetro que pueda describir la severidad de un movimiento sísmico ha sido constantemente estudiada desde los inicios de la ingeniería sísmica. Uno de los objetivos principales de dicho parámetro que se conoce como medida de intensidad sísmica, es estar íntimamente relacionado con la demanda sísmica, lo cual permite reducir las incertidumbres en la estimación de respuesta estructural de edificios. Algunas características deseables de dicha medida son su relación con la respuesta estructural y su capacidad para desacoplar las incertidumbres sismológicas y estructurales. Debido a la gran importancia que representa contar con una medida de $I S$ apropiada, se han desarrollado varios estudios orientados a encontrar un parámetro que refleje el potencial destructivo de un movimiento sísmico. Por ejemplo, en 1952 Housner propuso utilizar el área del espectro de pseudo-velocidad como medida de $I S$; algunos años después Von-Thun et al., (1988) sugirió el uso del área encerrada en el espectro de pseudoaceleración en un intervalo de periodos de 0.1 a $0.5 \mathrm{~s}$, para el análisis sísmico de presas. En los últimos años se han usado distintas medidas de intensidad sísmica, una de ellas es la aceleración máxima del suelo $\left(A_{m s}\right)$. Actualmente, la medida de intensidad más usada para el análisis de estructuras es la pseudoaceleración espectral medida en el periodo fundamental de vibración de la estructura de interés y se representa por $S a\left(T_{1}\right)$; sin embargo, se sabe que $S a\left(T_{1}\right)$ presenta algunas limitaciones cuando se usa como medida de $I S$, debido a que no considera el efecto de la elongación del periodo de vibración cuando ocurre comportamiento no lineal, ni tampoco incluye la contribución de modos superiores en la respuesta estructural.

Recientemente, otras medidas de intensidad más avanzadas han pretendido reducir las deficiencias de las medidas más comunes en la práctica de la ingeniería sísmica, las cuales se caracterizan por tener las siguientes propiedades: suficiencia y eficiencia. La suficiencia se refiere a que la respuesta estructural depende solamente de la medida de intensidad utilizada y no de las características sísmicas de la fuente, tales como la magnitud del sismo y la distancia al sitio de interés. Por otro lado, una medida de intensidad sísmica eficiente es aquella que presenta una buena relación con la respuesta estructural. Para cumplir con estas condiciones algunos investigadores sugieren utilizar medidas de intensidad sísmica vectorial, las cuales logran un mejor desempeño al incluir dos o más parámetros representativos del sismo (Baker y Cornell, 2005; Baker y Cornell, 2006; Bojórquez y Iervolino, 2011; Bojórquez et al., 2012a). Además, han mostrado mejorar la predicción de la respuesta estructural y consecuentemente la reducción de la dependencia de otras características de los movimientos sísmicos.

Baker y Cornell (2005) propusieron una medida de intensidad sísmica que consta de dos parámetros, la aceleración espectral en el periodo fundamental de vibración y épsilon $\varepsilon$ (donde $\varepsilon$ representa el número de desviaciones estándar que el logaritmo de una aceleración espectral observada difiere de la media logarítmica de la aceleración espectral obtenida a partir de una ley de atenuación). Con estos parámetros propusieron la medida de intensidad vectorial $\left\langle S a\left(T_{1}\right), \varepsilon\right\rangle$. Se ha encontrado que $\varepsilon$ describe la formal espectral; además, se ha demostrado que la forma espectral está íntimamente relacionada con la respuesta estructural. Sin embargo, Tothong y Luco (2007) mostraron la ineficacia de 
$\left\langle S a\left(T_{1}\right), \varepsilon\right\rangle$ como predictor de la respuesta estructural para el caso de registros de fuente cercana que manifiestan pulsos de velocidad, y propusieron una medida de $I S$ escalar avanzada basada en el desplazamiento espectral inelástico. Esta resulta adecuada para registros ordinarios y de fuente cercana, al menos en la predicción de la distorsión máxima de entrepiso, pero su aplicación es más compleja y no resulta tan adecuada para fines prácticos.

Shome (1999) y Baker y Cornell (2005) han demostrado las ventajas de utilizar medidas vectoriales de intensidad sísmica. Estos trabajos enfocan su atención en el incremento de la eficiencia (habilidad para predecir la respuesta estructural). Cabe mencionar que el uso de estas medidas suele ser poco práctico. Por lo que es más conveniente el empleo de medidas escalares de intensidad sísmica, ya que permiten un entendimiento más claro del potencial destructivo de un movimiento sísmico. Nótese que la evidencia existente muestra que los esfuerzos en plantear parámetros para definir una medida de $I S$ apropiada se concentra en la forma espectral debido a su relación con la respuesta estructural. Lo anterior indica que el primer paso es tratar de encontrar un parámetro capaz de representar mejor la forma espectral. Utilizando este razonamiento se ha propuesto recientemente una medida de $I S$ conocida como $I_{N p}$ (Bojórquez y Iervolino, 2011) cuyo objetivo es capturar con buena aproximación la forma espectral, a partir de un parámetro denominado $N_{p}$. Ellos observaron que medidas escalares y vectoriales basadas en $S a\left(T_{1}\right)$ y en la variable $N_{p}$, presentan una mejor relación con la respuesta sísmica no lineal de las estructuras. Por otro lado, en un estudio reciente Modica y Stafford (2014) concluyeron que la medida de intensidad sísmica vectorial más eficiente es aquella que se obtiene utilizando el parámetro $N_{p}$. Además, Buratti $(2011,2012)$ demostró el gran potencial de la medida propuesta por Bojórquez y Iervolino (2011) y su gran eficiencia, adicionalmente Buratti concluyó que una medida de intensidad sísmica eficiente resulta, al mismo tiempo, suficiente. A pesar de lo anterior, una limitación de $I_{N p}$ es que no toma en cuenta el efecto de los modos superiores en la respuesta estructural. Por esta razón, Bojórquez (2014) y Bojórquez et al., (2014) desarrollaron la medida de intensidad sísmica generalizada de Bojórquez $\left(I_{B}\right)$, la cual incorpora la influencia del comportamiento no lineal, de modos superiores, entre otras cosas.

En este trabajo se analizan un gran número de casos particulares de $I_{B}$, la cual se basa principalmente en la forma espectral a través del parámetro denominado $N_{p}$, donde normalmente la forma espectral más utiliza es en términos de la pseudo-aceleración, sin embargo otras formas espectrales también pueden utilizarse como lo señalan Bojórquez et al., (2011). Con la finalidad de mejorar la eficiencia de la medida de intensidad sísmica, en este estudio se utilizan otros parámetros tales como velocidad y desplazamiento. El objetivo primordial en este trabajo es encontrar cuales son los parámetros espectrales más útiles para definir $I_{B}$ de tal forma que tenga una relación más clara que otras medidas con el potencial destructivo de un movimiento sísmico, y a su vez con la respuesta estructural en términos de la distorsión máxima de entrepiso que es el principal parámetro empleado por los códigos de diseño sísmico para garantizar un desempeño estructural satisfactorio.

Para observar la importancia del parámetro $I_{B}$, y su relación con la respuesta estructural, se comparan un gran número de casos en que puede ser representada esta medida de intensidad; es decir, se confrontan varios casos particulares generados a partir de $I_{B}$, y se eligen aquellos que brindan los mejores resultados, los cuales se comparan con varias medidas de intensidad sísmica comúnmente utilizadas. En particular, se discute el uso del escalar $S a\left(T_{1}\right)$ que a pesar de ser uno de los parámetros más utilizados como medida de intensidad, resulta menos eficiente comparado tanto con $I_{N p}$ como con el parámetro analizado $I_{B}$. 


\section{MEDIDAS DE INTENSIDAD SÍSMICA}

Una medida de intensidad sísmica cuantifica las características de un movimiento sísmico que son importantes para describir su potencial destructivo, así como su capacidad para predecir la respuesta estructural (e.g. amplitud, frecuencia, duración del movimiento, etc). A continuación se hace una breve descripción de las medidas de intensidad sísmica utilizadas en el presente trabajo.

\section{Medida de intensidad $S a\left(T_{1}\right)$}

La medida de intensidad sísmica comúnmente usada por la mayoría de los ingenieros sísmicos, sismólogos y por códigos de diseño es la pseudo-aceleración espectral en el periodo fundamental de vibración de la estructura de interés, la cual fué previamente definida como $S a\left(T_{1}\right)$. Este parámetro es muy útil para predecir la respuesta de estructuras con poco comportamiento inelástico y gobernadas principalmente por su modo fundamental de vibración, esto se debe a la relación que tiene con la respuesta sísmica de tales estructuras, ya que es un parámetro que predice la respuesta sísmica de sistemas de un grado de libertad y sistemas de varios grados de libertad elásticos influenciados por el primer modo de manera bastante aproximada. Además, en algunos estudios se ha demostrado la suficiencia de $S a\left(T_{1}\right)$ con respecto a la magnitud y distancia (Shome, 1999; Iervolino y Cornell, 2005). Para estructuras donde las contribuciones de los modos superiores a la respuesta estructural es significativa, se ha demostrado que $S a\left(T_{1}\right)$ no ofrece buena precisión (Bazzurro y Cornell, 2002). Aún para estructuras cuya respuesta elástica es dominada por el primer modo de vibrar, se ha observado que $S a\left(T_{1}\right)$ no muestra buena relación con la respuesta de estructuras sometidas a sismos de fuente cercana (Luco, 2002). Por ello, el uso de $S a\left(T_{1}\right)$ puede conducir a distribuciones de demandas sísmicas con una gran variabilidad, particularmente en edificaciones sometidas a sismos de fuente cercana y de banda angosta (Bojórquez y Iervolino, 2011). Algunas limitaciones de $S a\left(T_{1}\right)$ es que por un lado no toma en consideración la elongación de los periodos en el rango inelástico, y por otro, este parámetro como medida de intensidad, no involucra efectos de modos superiores (periodos menores al fundamental) como se menciono anteriormente.

\section{Medida de intensidad $I_{N p}$}

A menudo las medidas de intensidad sísmica tratan de capturar la respuesta estructural a través de la forma espectral y cada una de ellas lo logra con diferente nivel de exactitud. Por ejemplo, $S a\left(T_{1}\right)$ predice con excelente aproximación la respuesta de sistemas elásticos de un grado de libertad o la respuesta de sistemas elásticos de múltiples grados de libertad dominados por el primer modo de vibrar $T_{l}$. Sin embargo, las estructuras presentan comportamiento no lineal cuando se someten a movimientos sísmicos intensos, y, en estos casos, sufrirán una elongación en su periodo de vibración, debido a que durante el movimiento cambiaran sus características dinámicas. Por lo tanto, el periodo efectivo de vibración será diferente al periodo fundamental elástico $T_{1}$, lo que implica que deben tomarse en cuenta las ordenadas espectrales para periodos mayores que $T_{l}$ como se ilustra en la figura 1. 


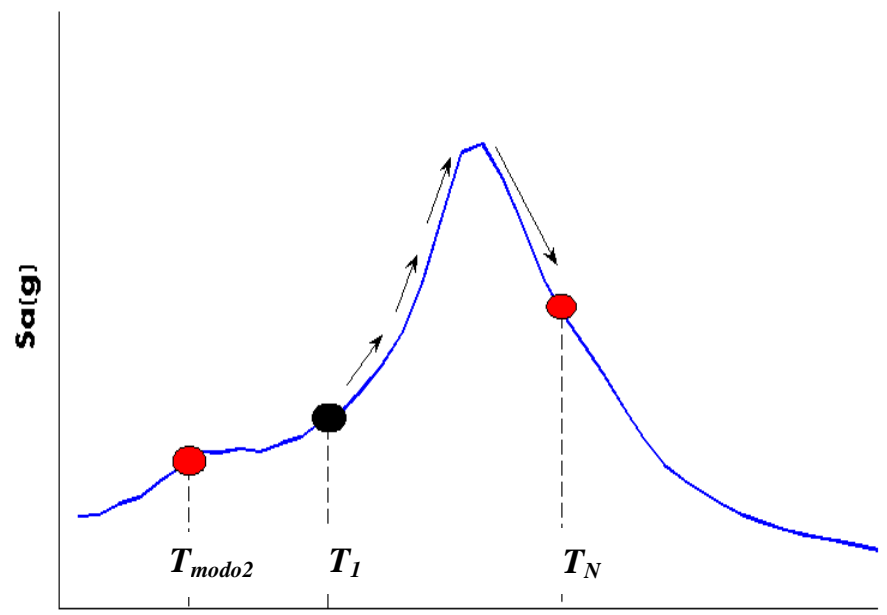

Periodo [s]

Figura 1. El periodo de la estructura $T_{l}$ muestra una elongación $\left(T_{N}\right)$ debido al comportamiento no lineal, $T_{\text {modo } 2}$ indica el periodo asociado al segundo modo de vibrar

Varias medidas de intensidad se han basado en representar con la mejor exactitud la forma espectral. Baker y Cornell (2006) propusieron utilizar la media geométrica de la aceleración espectral en un intervalo de periodos, $S a_{\text {avg }}\left(T_{1} \ldots T_{N}\right)$, con ello se pretendía evitar la influencia de picos y valles en la forma espectral, sin embargo, la figura 2 muestra dos espectros de respuesta con valores similares de $S a_{\text {avg }}\left(T_{1} \ldots T_{N}\right)$ y una forma espectral diferente. Lo anterior implica que ambos registros son totalmente distintos dentro del intervalo delimitado por $T_{1}$ y $T_{N}$, a pesar de tener un valor de $S a_{a v g}\left(T_{1} \ldots T_{N}\right)$ muy similar. Partiendo de esta observación, y para ser congruentes con la forma espectral Bojórquez y Iervolino (2011) sugieren normalizar $S a_{a v g}\left(T_{1} \ldots T_{N}\right)$ con respecto a $S a\left(T_{1}\right)$. De esta manera propusieron un nuevo parámetro denominado $N_{p}$, que se expresa en la ecuación 1.

$N_{p}=\frac{S a_{a v g}\left(T_{1} \ldots T_{N}\right)}{S a\left(T_{1}\right)}$

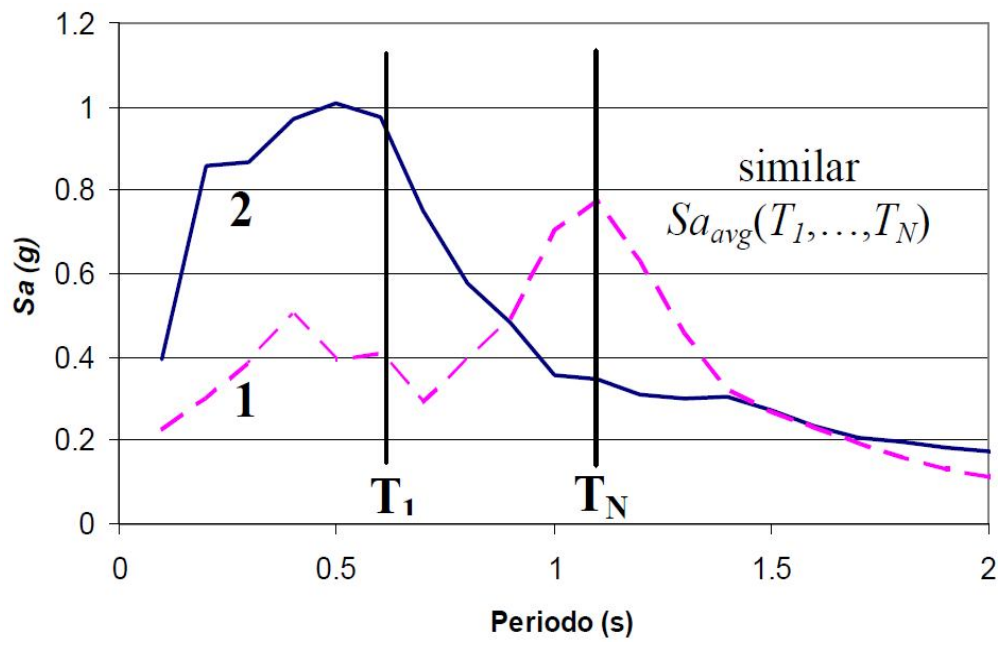

Figura 2. Espectro de respuesta de dos registros con un valor similar de $S a_{\text {avg }}\left(T_{1} \ldots T_{N}\right)$, que presentan formas espectrales distintas 
El término $N_{p}$ en la ecuación 1 se adoptó, debido a que representa la contribución de $\mathrm{N}$ puntos del espectro. Para entender mejor la información dada por $N_{p}$, supongamos que tenemos uno o un número $n$ de registros con un valor promedio de $N_{p}$ cercano a uno, esto indica que se espera que el espectro promedio sea plano en el intervalo de periodos comprendido entre $T_{l}$ y $T_{N}$. Para un valor de $N_{p}$ menor a la unidad el espectro promedio tendrá una pendiente negativa. Como ejemplo, el valor medio de $N_{p}$ para un grupo de registros en el intervalo de periodos $T_{l}=0.6 \mathrm{~s}$ hasta $T_{N}=2 T_{l}$ (ver figura 3a) es 0.39 . En el caso de valores de $N_{p}$ mayores que la unidad, éstos estarán asociados a una zona de incremento en las ordenadas espectrales, y tendrán una pendiente positiva a partir de $T_{1}$. En la figura $3 \mathrm{~b}$, se aprecia que el espectro promedio, para un conjunto de registros de banda angosta, con valor medio de $N_{p}=1.9$ en un intervalo de periodos $T_{l}=1.2 \mathrm{~s}$ a $T_{N}=2 T_{1}$, muestra un incremento en sus aceleraciones espectrales.

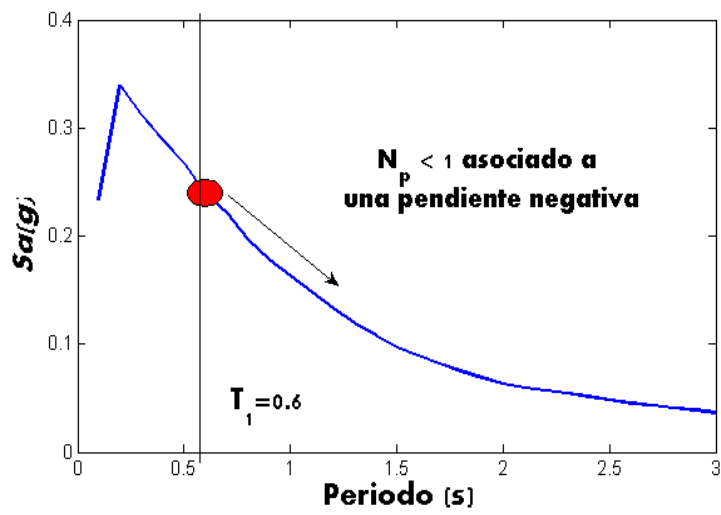

a)

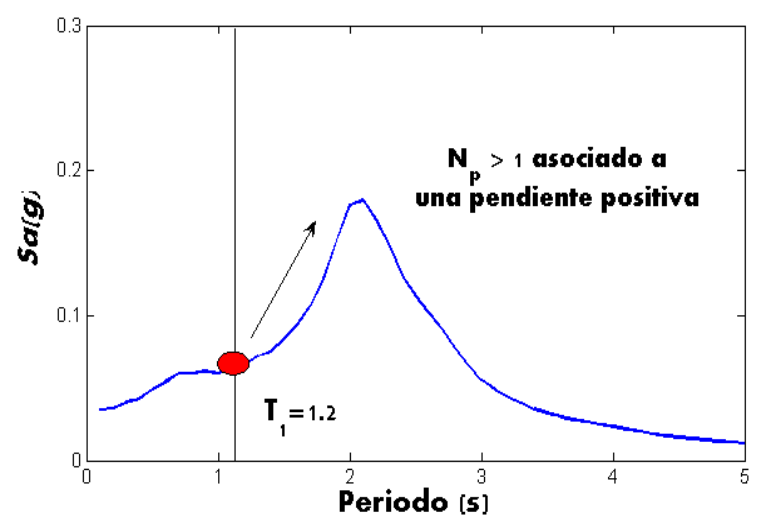

b)

Figura 3. Espectro de respuesta promedio para un grupo de: a) registros ordinarios con $N p=0.39$, b) registros de banda angosta con $N p=1.9$

Finalmente, la normalización con respecto a $S a\left(T_{1}\right)$ permite que $N_{p}$ sea independiente del nivel de escalamiento de los registros basados en $S a\left(T_{l}\right)$, pero lo más importante de esta normalización es que ayuda a mejorar el conocimiento sobre el curso que lleva el espectro en el intervalo de periodos $T_{1}$ y $T_{N}$, el cual está relacionado con la respuesta no lineal de la estructura.

Las medidas escalares tradicionales de intensidad sísmica (e.g. $S a\left(T_{1}\right), A_{m s}$ ) tienen ciertas desventajas como la insuficiencia o limitada eficiencia, las cuales han sido superadas por otras medidas de intensidad sísmica tanto escalaras como vectoriales. Una medida de intensidad sísmica vectorial basada en $S a\left(T_{1}\right)$ y en combinación con el parámetro $N_{p}$ ya ha sido investigada y se describirá enseguida. Bojórquez et al., (2008) han comparado el vector $\left\langle S a, N_{p}\right\rangle$ con otras medidas de intensidad y encontraron que esta medida de intensidad representa un avance en la predicción de respuesta sísmica en términos de ductilidad, energía histerética disipada e índice de daño de Park y Ang (Park y Ang 1985) para estructuras sometidas a registros ordinarios, de banda angosta y de fuente cercana. Bojórquez y Iervolino (2011) y Bojórquez et al., (2012a) han verificado que el vector es consistente, lo que significa que cuando los registros se escalan para valores similares de $S a\left(T_{1}\right)$, la respuesta sísmica dependerá del valor de $N_{p}$; el vector es relativamente eficiente independientemente de las características de los movimientos sísmicos (e.g. ordinarios, de fuente cercana y de banda angosta) o de los parámetros de demanda sísmica considerados. Finalmente, existe buena relación entre los valores de $N_{p}$ y la forma espectral en un amplio intervalo de periodos.

El vector $\left\langle S a, N_{p}\right\rangle$ resulta mucho más eficiente que las medidas de intensidad frecuentemente utilizadas. Sin embargo, el uso de una medida de intensidad vectorial con la cual se pretenda desarrollar análisis probabilístico de la demanda sísmica de una estructura, requerirá la estimación de distribuciones 
condicionales de $N_{p}$ dada $S a\left(T_{1}\right)$. Por otro lado, el análisis probabilístico de la demanda sísmica se facilita por medio del uso de medidas de intensidad escalar, y la relación con la respuesta estructural también se vuelve más clara. Por esta razón, Bojórquez y Iervolino (2011) propusieron una medida de intensidad sísmica escalar basada en los parámetros $S a\left(T_{1}\right)$ y $N_{p}$ con características similares a la medida de intensidad propuesta por Córdova et al., (2001), la cual se define de la siguiente manera:

$I_{N p}=S a\left(T_{1}\right) \cdot N_{p}^{\alpha}$

En la ecuación 2, $I_{N p}$ es la medida de intensidad sísmica escalar, y el valor $\alpha$ es un parámetro que debe ser calibrado, en función de la estructura y el parámetro de demanda sísmica seleccionado. Buratti $(2011,2012)$ demostró que $I_{N p}$ resulta más eficiente que un gran de número de medidas de intensidad sísmica encontradas en la literatura, entre las cuales se encuentran las propuestas por Housner (1952, 1970, 1975), Housner y Jennings (1964), Arias (1970), Araya y Saragoni (1980), Aptikaev (1982), Sarma y Yang (1987), FEMA (1994), Shome et al., (1998), Shome (1999), Mehanny y Deierlein (2000), Cordova et al., (2001), Riddell y Garcia (2001), Kurama y Farrow (2003), Baker y Cornell (2004), Baker y Cornell (2005), Baker y Cornell (2006), Mehanny (2009), entre otras.

En la expresión 2 se observa lo siguiente: a) la aceleración espectral en el primer modo de vibrar o el periodo de la estructura es un caso particular de $I_{N p}$, y este ocurre cuando $\alpha$ es igual a cero; b) la media geométrica $S a_{\text {avg }}\left(T_{1} \ldots T_{N}\right)$ representa el caso particular de $I_{N p}$ cuando $\alpha=1$; y c) la medida de intensidad vectorial propuesta por Córdova et al., (2001), denominada $\left\langle S a, R_{T 1, T 2}\right\rangle$, también corresponde a un caso particular cuando solo dos puntos del espectro son tomados en consideración, por ejemplo, $T_{l}$ y $T_{N}$. Bojórquez y Iervolino (2011) sugieren que los valores óptimos de $\alpha$ están en un intervalo que va desde cero hasta uno, lo cual significa dotar a las ordenadas espectrales con un nivel diferente de importancia cuando se analice $I_{N p}$ más allá del primer modo.

\section{Medida de intensidad generalizada de Bojórquez $I_{B}$}

A pesar de que $I_{N p}$ ha resultado más eficiente en la predicción de la respuesta estructural comparado con otros parámetros, una de sus limitaciones es la falta de consideración del efecto de los modos superiores, ya que no incluye ordenadas espectrales asociadas a periodos inferiores al periodo fundamental de la estructura. Con la finalidad de mejorar la capacidad predictora de $I_{N p}$, Bojórquez propuso una medida de intensidad sísmica generalizada basada en el parámetro $N_{p}$, el uso de la forma espectral. La característica principal de este parámetro es que un número importante de medidas de intensidad son un caso particular de esta medida de intensidad. En el presente estudio se analizan un número determinado de casos particulares de la intensidad de Bojórquez, esto es, cuando de utiliza los espectros de pseudoaceleración, velocidad y desplazamiento, en donde es posible utilizar cada parámetro espectral por separado o combinar estos parámetros espectrales para generar casos particulares híbridos de $I_{B}$, para el caso general la medida puede expresarse como se indica en la ecuación 3. Note que en esta expresión es posible utilizar cualquier forma espectral tales como energía de entrada o parámetros inelásticos, y no solo las formas espectrales seleccionadas en el presente estudio.

$$
I_{B}=S\left(T_{1}\right)^{\alpha_{1}} \cdot N_{p}^{\alpha_{2}} \cdot \prod_{i=2}^{i=\# \text { modos }}\left[R_{(T 1, T m o d o i)}^{\alpha_{3(\bmod o)}}\right]
$$

En la ecuación 3, $I_{B}$ representa la intensidad de Bojórquez; $S\left(T_{1}\right)$ representa un parámetro espectral tomado de cualquiera de los espectros de pseudo-aceleración, velocidad y/o desplazamiento; $N_{p}$ de la ecuación 1 por conveniencia lo podemos reescribir como se indica en la ecuación $4 ; R_{T 1, T m o d o i}$ se define como el cociente de un parámetro espectral en el periodo del i-ésimo modo de vibrar de la estructura 
$\left(S\left(T_{\text {modoi }}\right)\right)$ y un parámetro espectral en el periodo fundamental de vibración $\left(S\left(T_{1}\right)\right)$, siendo $T_{1}$ mayor que $T_{\text {modoi }}$ (ver ecuación 5).

$$
\begin{gathered}
N_{p}=\frac{S_{\text {avg }}\left(T_{1} \ldots T_{N}\right)}{S\left(T_{1}\right)} \\
R_{(T 1, \text { Tmodoi })}=\frac{S\left(T_{\text {modoi }}\right)}{S\left(T_{1}\right)}
\end{gathered}
$$

La ecuación 3 muestra que el parámetro $I_{B}$ incorpora tanto la predicción del comportamiento no lineal como el efecto de los modos superiores. Note que $I_{N p}$ es un caso particular de $I_{B}$ cuando se utiliza el espectro de pseudo-aceleración como parámetro espectral y $\alpha_{3(\text { modoi })}$ es igual a cero para todos los modos. Además, en esta expresión los exponentes $\alpha_{1}, \alpha_{2}$ y $\alpha_{3}$ son parámetros que deben ser calibrados. Es importante mencionar que el exponente $\alpha_{3(\text { modoi) }}$ asociado a un modo de vibrar puede variar para cada modo, por simplicidad en la ecuación 3 se supone igual para todos los modos y se puede reescribir de la siguiente manera:

$I_{B}=S\left(T_{1}\right)^{\alpha_{1}} \cdot N_{p}^{\alpha_{2}} \cdot \prod_{i=2}^{i=\# \text { modes }}\left[R_{(T 1, T m o d o i)}^{\alpha_{3}}\right]$

\section{METODOLOGÍA}

\section{Modelos estructurales}

Las estructuras analizadas en este trabajo consisten en edificaciones formadas a base de marcos de acero de 4, 6, 8, 10, 14 y 18 niveles, cuyas características geométricas se muestran en la figura 4 y 5 . Se consideró que los marcos están ubicados en la Zona III-b del valle de México de acuerdo al Reglamento de Construcciones del Distrito Federal (RCDF, 2004). Cabe mencionar que en dicha zona ocurrieron la mayor cantidad de daños debido al sismo del 19 de septiembre de 1985. Se supone que el uso de las edificaciones es para oficinas, por lo cual se consideran estructuras tipo B. Para el análisis de los marcos se utilizó el programa RUAUMOKO (Carr, 2008), los cuales fueron modelados considerando que la base se encuentra empotrada y tomando en cuenta efectos de segundo orden. Las vigas y las columnas se modelaron como elementos de marco los cuales concentran su respuesta inelástica en los extremos. Se consideró que los elementos tienen un comportamiento bilineal con $3 \%$ de rigidez de posfluencia. Las características dinámicas principales de las edificaciones se muestran en la tabla 1 , en la cual $T_{l}$ representa el periodo fundamental de vibración de la estructura, $T_{\text {modo2 }}$ el periodo correspondiente al segundo modo de vibrar y finalmente el coeficiente sísmico se expresa como $C_{y}$.

Tabla 1. Características dinámicas de los marcos

\begin{tabular}{cccc}
\hline \multirow{2}{*}{ Marco estructural } & \multicolumn{2}{c}{ Periodos de vibración $\mathbf{( s )}$} & \multirow{2}{*}{$\boldsymbol{C}_{\boldsymbol{y}}$} \\
\cline { 2 - 3 } & $\boldsymbol{T}_{\mathbf{1}}$ & $\boldsymbol{T}_{\text {modo2 }}$ & 0.45 \\
4 niveles & 0.90 & 0.27 & 0.42 \\
6 niveles & 1.07 & 0.34 & 0.38 \\
8 niveles & 1.20 & 0.39 & 0.36 \\
10 niveles & 1.35 & 0.45 & 0.25 \\
14 niveles & 1.91 & 0.65 & 0.185 \\
18 niveles & 2.53 & 0.87 &
\end{tabular}



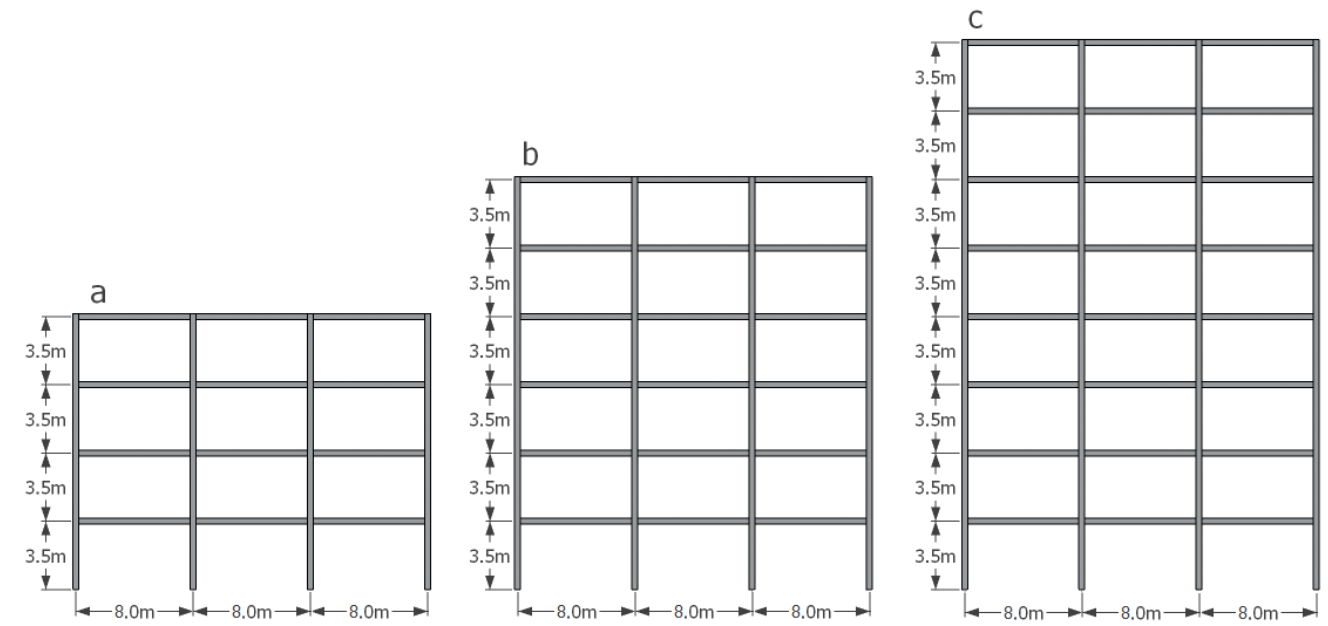

Figura 4. Características geométricas de los marcos de acero: a) 4 niveles, b) 6 niveles y c) 8 niveles
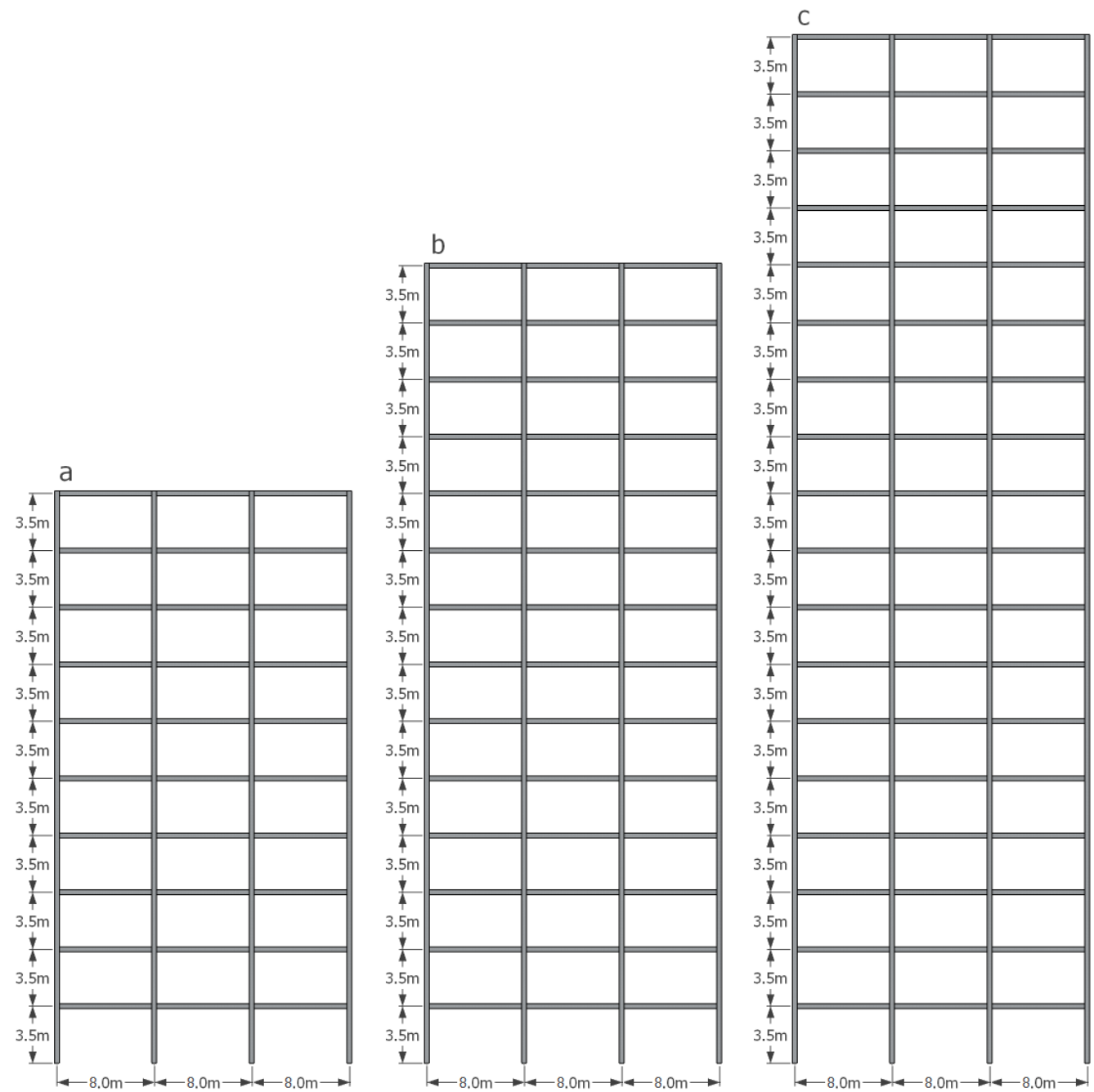

Figura 5. Características geométricas de los marcos de acero: a) 10 niveles, b) 14 niveles y c) 18 niveles 


\section{Registros sísmicos utilizados}

Para analizar las características de las medidas de intensidad sísmicas antes presentadas, se seleccionaron dos conjuntos de registros sísmicos. El primer grupo de movimientos sísmicos se tomaron de la base de datos del Centro de Investigación de Ingeniería Sísmica del Pacifico (Base de Datos NGA). Los acelerogramas son representativos de suelos tipo A, B, C, D, y E, y obtenidos de eventos sísmicos con magnitudes que van desde 6.0 hasta 7.9, y registrados a distancias de Joyner-Boore $\left(d_{J B}\right)$ desde 1 hasta 365 $\mathrm{km}$ (definida como la distancia horizontal más corta desde el sitio donde se registró el movimiento hasta la proyección vertical de la falla). La figura 6 muestra la distribución de magnitud y distancia de los eventos considerados. El tipo de suelo se define por la clasificación de Geomatrix (Geomatrix's Classification), que se basa en las características subyacentes geotécnicas del suelo, conocida como GMX3. Dentro de la clasificación indicada, mientras que el suelo tipo A representa una roca sana el suelo tipo E es representativo de suelos blandos, con velocidades de ondas de cortante promedio menores a $150 \mathrm{~m} / \mathrm{s}$. Las características de los registros sísmicos utilizados se listan en las tablas 2 a 6.

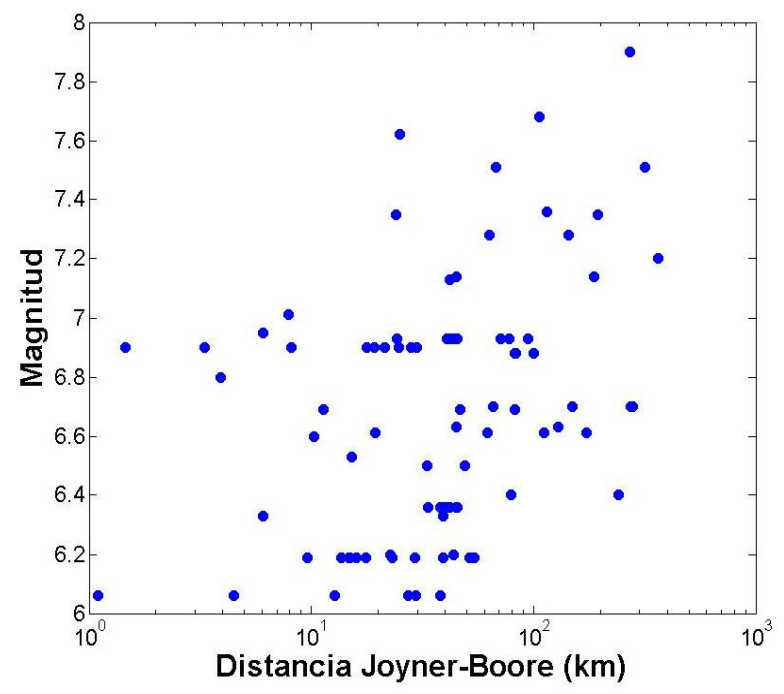

Figura 6. Distribución del primer conjunto de registros seleccionado

Tabla 2. Conjunto de registros sísmicos correspondiente a suelo tipo A

\begin{tabular}{cccccc}
\hline No. Registro & Nombre del sismo & EQID & Nombre de la estación & Magnitud & $\boldsymbol{d}_{\mathbf{J B}}(\mathbf{k m})$ \\
\hline 1 & Helena, Montana-01 & 0001 & Carroll College & 6.00 & ND \\
2 & Parkfield & 0025 & Temblor pre-1969 & 6.19 & 15.96 \\
3 & San Fernando & 0030 & Carbon Canyon Dam & 6.61 & 61.79 \\
4 & Gazli, USSR & 0041 & Karakyr & 6.80 & 3.92 \\
5 & Tabas, Iran & 0046 & Dayhook & 7.35 & 0.00 \\
6 & Imperial Valley-06 & 0050 & Cerro Prieto & 6.53 & 15.19 \\
7 & Irpinia, Italy-01 & 0068 & Bagnoli Irpinio & 6.90 & 8.14 \\
8 & Coalinga-01 & 0076 & Parkfield - Cholame 3E & 6.36 & 40.01 \\
9 & Taiwan SMART1(25) & 0086 & SMART1 E02 & 6.50 & ND \\
10 & Borah Peak, ID-01 & 0087 & ANL-768 Power Plant & 6.88 & 100.22 \\
11 & Morgan Hill & 0090 & Corralitos & 6.19 & 23.23 \\
12 & Nahanni, Canada & 0097 & Site 2 & 6.76 & 0.00 \\
13 & N. Palms Springs & 0101 & Anza - Red Mountain & 6.06 & 38.22 \\
14 & Chalfant Valley-02 & 0103 & Bishop -Paradise Lodge & 6.19 & 14.97 \\
15 & Taiwan SMART1(45) & 0109 & SMART1 E02 & 7.30 & ND \\
16 & Loma Prieta & 0118 & APEEL 7 - Pulgas & 6.93 & 41.68 \\
17 & Landers & 0125 & Big Tujunga, Angeles Na & 7.28 & 144.13 \\
18 & Big Bear-01 & 0126 & LA - City Terrace & 6.46 & ND \\
19 & Northridge-01 & 0127 & Antelope Buttes & 6.69 & 46.65 \\
20 & Sitka, Alaska & 0140 & Juneau Fisheries Lab & 7.68 & 106.71 \\
\hline
\end{tabular}


Tabla 3. Conjunto de registros sísmicos correspondiente a suelo tipo B

\begin{tabular}{cccccc}
\hline No. Registro & Nombre del sismo & EQID & Nombre de la estación & Magnitud & $\boldsymbol{d}_{\mathbf{J B}}(\mathbf{k m})$ \\
\hline 1 & Southern Calif & 0014 & San Luis Obispo & 6.00 & ND \\
2 & Borrego Mtn & 0028 & San Onofre - So Cal & 6.63 & 129.11 \\
3 & Parkfield & 0025 & Cholame - Shandon & 6.19 & 9.58 \\
4 & San Fernando & 0030 & Castaic - Old Ridge & 6.61 & 19.33 \\
5 & Friuli, Italy-01 & 0040 & Barcis & 6.50 & 49.13 \\
6 & Irpinia, Italy-01 & 0068 & Bisaccia & 6.90 & ND \\
7 & Coalinga-01 & 0076 & Parkfield - Cholame 2E & 6.36 & 41.99 \\
8 & Borah Peak, ID-01 & 0087 & CPP-601 & 6.88 & 82.60 \\
9 & Morgan Hill & 0090 & Gilroy - Gavilan Coll. & 6.19 & 14.83 \\
10 & N. Palms Springs & 0101 & Cranston Forest Station & 6.06 & 27.21 \\
11 & Duzce, Turkey & 0138 & Sakarya & 7.14 & 45.16 \\
12 & Northridge-02 & 0147 & Castaic - Old Ridge & 6.05 & ND \\
13 & Hector Mine & 0158 & Amboy & 7.13 & 41.82 \\
14 & Nenana Mountain & 0168 & Fairbanks - Geophysic. & 6.70 & 148.82 \\
15 & Coalinga-01 & 0076 & Parkfield - Cholame 4W & 6.36 & 45.49 \\
16 & Coalinga-01 & 0076 & Parkfield - Fault Zone 4 & 6.36 & 33.42 \\
17 & Coalinga-01 & 0076 & Parkfield - Gold Hill 3W & 6.36 & 38.10 \\
18 & Borah Peak, ID-02 & 0087 & CPP-610 & 6.88 & 83.00 \\
19 & Morgan Hill & 0090 & Capitola & 6.19 & 39.08 \\
20 & N. Palm Springs & 0101 & Hurkey Creek Park & 6.06 & 29.56 \\
\hline
\end{tabular}

Tabla 4. Conjunto de registros sísmicos correspondiente a suelo tipo $\mathrm{C}$

\begin{tabular}{cccccc}
\hline No. Registro & Nombre del sismo & EQID & Nombre de la estación & Magnitud & $\boldsymbol{d}_{\boldsymbol{J B}}(\mathbf{k m})$ \\
\hline 1 & San Fernando & 0030 & Anza Post Office & 6.61 & 173.16 \\
2 & Tabas, Iran & 0046 & Boshrooyeh & 7.35 & 24.07 \\
3 & Mammoth Lakes-01 & 0056 & Convict Creek & 6.06 & 1.1 \\
4 & Victoria, Mexico & 0064 & SAHOP Casa Flores & 6.33 & 39.1 \\
5 & Trinidad & 0067 & Rio Dell Overpass - FF & 7.20 & ND \\
6 & Irpinia, Italy-01 & 0068 & Mercato San Severino & 6.90 & 29.79 \\
7 & Irpinia, Italy-02 & 0069 & Rionero In Vulture & 6.20 & 22.68 \\
8 & Corinth, Greece & 0072 & Corinth & 6.60 & 10.27 \\
9 & Coalinga-01 & 0076 & Parkfield - Cholame 3W & 6.36 & 44.82 \\
10 & lerissos, Greece & 0083 & lerissos & 6.70 & 65.67 \\
11 & N. Palm Springs & 0101 & Fun Valley & 6.06 & 12.79 \\
12 & Chalfant Valley-02 & 0103 & Convict Creek & 6.19 & 29.35 \\
13 & Loma Prieta & 0118 & APEEL 9 & 6.93 & 40.85 \\
14 & Cape Mendocino & 0123 & Rio Dell Overpass - FF & 7.01 & 7.88 \\
15 & Landers & Fort Irwin & 7.28 & 62.98 \\
16 & Big Bear-01 & 0125 & Featherly Park - Maint & 6.46 & ND \\
17 & Northridge-01 & 0126 & Canyon Country - W & 6.69 & 11.39 \\
18 & Kozani, Greece-01 & 0130 & Kardista & 6.40 & 79.33 \\
19 & Dinar, Turkey & 0134 & Bursa & 6.40 & 240.71 \\
20 & Gulf of Aqaba & 0135 & Hadera & 7.20 & 365.14 \\
\hline
\end{tabular}


Tabla 5. Conjunto de registros sísmicos correspondiente a suelo tipo D

\begin{tabular}{cccccc}
\hline No. Registro & Nombre del sismo & EQID & Nombre de la estación & Magnitud & $\boldsymbol{d}_{\mathbf{J B}}(\mathbf{k m})$ \\
\hline 1 & Imperial Valley-02 & 0006 & El Centro Array \#9 & 6.95 & 6.09 \\
2 & Northwest Calif-02 & 0007 & Ferndale City Hall & 6.60 & ND \\
3 & Borrego & 0009 & El Centro Array \#9 & 6.50 & ND \\
4 & Kern County & 0012 & LA - Hollywood Stor FF & 7.36 & 114.62 \\
5 & El Alamo & 0019 & El Centro Array \#9 & 6.80 & ND \\
6 & Parkfield & 0025 & Cholame - Shandon & 6.19 & 17.64 \\
7 & Borrego Mtn & 0028 & El Centro Array \#9 & 6.63 & 45.12 \\
8 & San Fernando & 0030 & Bakersfield - Harvey. & 6.61 & 111.88 \\
9 & Friuli, Italy-01 & 0040 & Codroipo & 6.50 & 33.32 \\
10 & Tabas, Iran & 0046 & Kashmar & 7.35 & 193.91 \\
11 & Imperial Valley-06 & 0050 & Bonds Corner & 6.53 & 0.47 \\
12 & Mammoth Lakes-01 & 0056 & Mammoth Lakes H. S. & 6.06 & 4.48 \\
13 & Victoria, Mexico & 0064 & Victoria Hospital Sotano & 6.33 & 6.07 \\
14 & Irpinia, Italy-02 & 0069 & Bovino & 6.20 & 43.5 \\
15 & Morgan Hill & 0090 & Gilroy Array \#2 & 6.19 & 13.68 \\
16 & Loma Prieta & 0118 & Agnews State Hospital & 6.93 & 24.27 \\
17 & Georgia, USSR & 0120 & Baz & 6.20 & ND \\
18 & Northridge-01 & 0127 & Newport Bch - Newp. & 6.69 & 82.03 \\
19 & Kocaeli, Turkey & 0136 & Bornova & 7.51 & 315.9 \\
20 & Chi-Chi, Taiwan & 0137 & CHY002 & 7.62 & 24.98 \\
\hline
\end{tabular}

Tabla 6. Conjunto de registros sísmicos correspondiente a suelo tipo E

\begin{tabular}{cccccc}
\hline No. Registro & Nombre del sismo & EQID & Nombre de la estación & Magnitud & $\boldsymbol{d}_{\boldsymbol{J B}}(\mathbf{k m})$ \\
\hline 1 & Loma Prieta & 0118 & APEEL 2 - Redwood City & 6.93 & 43.06 \\
2 & Kobe, Japan & 0129 & OSAJ & 6.90 & 21.35 \\
3 & Kocaeli, Turkey & 0136 & Ambarli & 7.51 & 68.09 \\
4 & Duzce, Turkey & 0138 & Ambarli & 7.14 & 187.99 \\
5 & Nenana Mountain & 0168 & Anchorage - DOI Off. & 6.70 & 272.87 \\
6 & Denali, Alaska & 0169 & Anchorage - DOI Off. & 7.90 & 272.51 \\
7 & Kobe, Japan & 0129 & Shin-Osaka & 6.90 & 19.14 \\
8 & Loma Prieta & 0118 & Treasure Island & 6.93 & 77.32 \\
9 & Loma Prieta & 0118 & Larkspur Ferry Terminal & 6.93 & 94.56 \\
10 & Loma Prieta & 0118 & Foster City - APEEL 1 & 6.93 & 43.77 \\
11 & Morgan Hill & 0090 & Foster City - APEEL 1 & 6.19 & 53.89 \\
12 & Morgan Hill & 0090 & APEEL 1E - Hayward & 6.19 & 51.68 \\
13 & Loma Prieta & 0118 & Alameda Naval AiR Stn & 6.93 & 70.9 \\
14 & Loma Prieta & 0118 & Foster City - Menhaden & 6.93 & 45.42 \\
15 & Kobe, Japan & 0129 & Fukushima & 6.9 & 17.85 \\
16 & Kobe, Japan & 0129 & Morigawachi & 6.9 & 24.78 \\
17 & Kobe, Japan & 0129 & Port Island (0 m) & 6.9 & 3.31 \\
18 & Kobe, Japan & 0129 & Sakai & 6.9 & 28.08 \\
19 & Kobe, Japan & 0129 & Takatori & 6.90 & 1.46 \\
20 & Nenana Mountain & 0168 & Anchorage - K2-15 & 6.70 & 279.32 \\
\hline
\end{tabular}

Para simular el comportamiento de las estructuras en suelos muy blandos característicos de la Ciudad de México (Suelo MB) o sismos de banda angosta, se utilizaron los registros sísmicos listados en la tabla 7. Los movimientos de banda angosta se caracterizan por afectar considerablemente estructuras que tienen periodos de vibración dentro de cierto intervalo, especialmente aquellas que sufren del efecto de ablandamiento. De hecho, estos registros demandan una gran cantidad de energía en las estructuras en comparación con los movimientos de banda ancha (Terán-Gilmore y Jirsa, 2007).

En este estudio se usaron 30 registros obtenidos en el suelo blando del Valle de México. La magnitud de estos varía desde 6.1 hasta 8.1, con distancias epicentrales que van desde 286 hasta $541 \mathrm{~km}$ (ver tabla 7). 
Tabla 7. Conjunto de registros sísmicos correspondiente a suelo muy blando

\begin{tabular}{|c|c|c|c|c|c|}
\hline No. Registro & Nombre del sismo & Fecha & Nombre de la estación & Magnitud & Dist. Epicentral (km) \\
\hline 1 & Michoacán & $19 / 09 / 85$ & SCT & 8.1 & 366 \\
\hline 2 & Michoacán & $21 / 09 / 85$ & Tlahuac deportivo & 7.6 & 323 \\
\hline 3 & Guerrero & $25 / 04 / 89$ & Alameda & 6.9 & 293 \\
\hline 4 & Guerrero & $25 / 04 / 89$ & Garibaldi & 6.9 & 294 \\
\hline 5 & Guerrero & $25 / 04 / 89$ & SCT & 6.9 & 289 \\
\hline 6 & Guerrero & $25 / 04 / 89$ & Sector Popular & 6.9 & 286 \\
\hline 7 & Guerrero & $25 / 04 / 89$ & Tlatelolco TL08 & 6.9 & 295 \\
\hline 8 & Guerrero & $25 / 04 / 89$ & Tlatelolco TL55 & 6.9 & 293 \\
\hline 9 & Oaxaca. - Guerrero & $14 / 09 / 95$ & Alameda & 7.3 & 303 \\
\hline 10 & Oaxaca. - Guerrero & $14 / 09 / 95$ & Garibaldi & 7.3 & 303 \\
\hline 11 & Oaxaca. - Guerrero & $14 / 09 / 95$ & Liconsa & 7.3 & 286 \\
\hline 12 & Oaxaca. - Guerrero & $14 / 09 / 95$ & Plutarco Elías Calles & 7.3 & 298 \\
\hline 13 & Oaxaca. - Guerrero & $14 / 09 / 95$ & Sector Popular & 7.3 & 295 \\
\hline 14 & Oaxaca. - Guerrero & $14 / 09 / 95$ & Tlatelolco TL08 & 7.3 & 304 \\
\hline 15 & Oaxaca. - Guerrero & $14 / 09 / 95$ & Tlatelolco TL55 & 7.3 & 303 \\
\hline 16 & Colima & 09/10/95 & Cibeles & 7.5 & 536 \\
\hline 17 & Colima & 09/10/95 & CU Juárez & 7.5 & 537 \\
\hline 18 & Colima & 09/10/95 & C. urbano P. Juárez & 7.5 & 537 \\
\hline 19 & Colima & 09/10/95 & Córdoba & 7.5 & 537 \\
\hline 20 & Colima & 09/10/95 & Liverpool & 7.5 & 537 \\
\hline 21 & Colima & 09/10/95 & Plutarco Elías Calles & 7.5 & 539 \\
\hline 22 & Colima & 09/10/95 & Sector Popular & 7.5 & 540 \\
\hline 23 & Colima & 09/10/95 & Valle Gómez & 7.5 & 541 \\
\hline 24 & Michoacán & $11 / 01 / 97$ & CU Juárez & 6.9 & 379 \\
\hline 25 & Michoacán & $11 / 01 / 97$ & C. urbano P. Juárez & 6.9 & 379 \\
\hline 26 & Michoacán & $11 / 01 / 97$ & García Campillo & 6.9 & 381 \\
\hline 27 & Michoacán & $11 / 01 / 97$ & Plutarco Elías Calles & 6.9 & 381 \\
\hline 28 & Michoacán & $11 / 01 / 97$ & Est. \# 10 Roma A & 6.9 & 380 \\
\hline 29 & Michoacán & $11 / 01 / 97$ & Est. \# 11 Roma B & 6.9 & 380 \\
\hline 30 & Michoacán & $11 / 01 / 97$ & Tlatelolco TL08 & 6.9 & 383 \\
\hline
\end{tabular}

Estos dos conjuntos de registros fueron considerados para representar movimientos sísmicos registrados en todo tipo de suelo. Por lo tanto, los movimientos sísmicos representan diferentes formas espectrales. Esto permitirá demostrar el potencial de las medidas de intensidad sísmica utilizadas para predecir el comportamiento de edificaciones sometidas a registros con características muy diversas y provenientes de distinta fuente.

\section{Estimación de la eficiencia de las medidas de intensidad sísmica}

Se escalaron 100 registros sísmicos para suelos tipo A, B, C, D y E, y los 30 registros característicos de la zona blanda de la Ciudad de México, para distintos valores de la aceleración espectral, que van desde $0.5 \mathrm{~g}$ hasta $1.2 \mathrm{~g}$. No se utilizaron valores menores a 0.5 debido a que en el intervalo de intensidades que va desde cero hasta $0.5 \mathrm{~g}$ no existe presencia de no linealidad en las edificaciones o es despreciable.

El primer paso es efectuar un análisis dinámico incremental (Vamvatsikos y Cornell, 2002) con los registros sísmicos escalados para distintos niveles de intensidad usando el programa RUAUMOKO. Como resultado de este análisis se obtuvieron las distorsiones máximas de entrepiso $(\gamma)$ para cada estructura y tipo de suelo. Se seleccionó este parámetro de desempeño debido a que es el más utilizado por los códigos de diseño sísmico para garantizar un comportamiento sísmico satisfactorio de las edificaciones. En el presente trabajo se utiliza la notación MB iniciales de muy blando para definir este tipo de suelo.

Posteriormente se calcula el logaritmo natural de la distorsión máxima de entrepiso y también el logaritmo natural de la medida de intensidad utilizada. La figura 7 muestra los resultados para el caso de $S a\left(T_{1}\right)$. En el siguiente paso se realiza un análisis de regresión lineal para todos los niveles de escalamiento, primeramente para $S a\left(T_{1}\right)$ y además se obtiene la desviación estándar del logaritmo natural de la distorsión máxima de entrepiso $\left(\sigma_{\ln (\gamma)}\right)$. Para estimar $\sigma_{\ln (\gamma)}$ usando $I_{N p}$ así como todos los casos 
particulares en estudio de $I_{B}$, se realiza el análisis de regresión considerando en el eje horizontal el logaritmo natural de ambos parámetros.

Una vez obtenida la desviación estándar para $S a\left(T_{1}\right), I_{N p}$ y todos los casos particulares en estudio de $I_{B}$, se procede a realizar una comparación en una primera instancia entre los casos particulares en estudio de $I_{B}$ para determinar cuáles son los tres casos que presenta la menor desviación estándar, una vez encontrados estos casos se procede a comparar dichos casos con las otras dos medidas de intensidad sísmica, para determinar cuál de las tres medidas intensidad sísmica es la más eficiente, se busca aquella que tenga la menor desviación estándar, ya que el objetivo es minimizar la desviación estándar debido a que una medida de intensidad es eficiente cuando se reduce la incertidumbre asociada a la respuesta estructural.

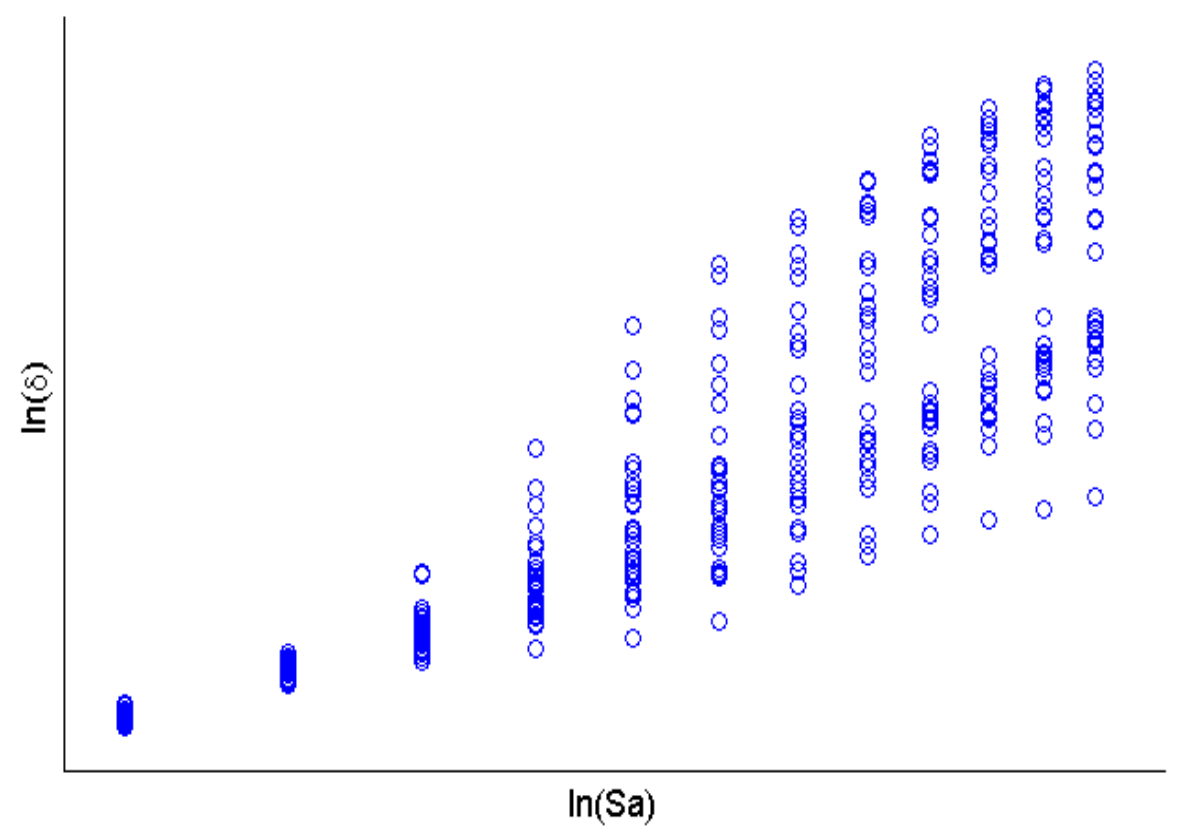

Figura 7. Logaritmo natural de $S a\left(T_{1}\right)$ versus el logaritmo natural de la distorsión máxima de entrepiso

\section{Casos particulares en estudio de $I_{B}$}

Para generar los casos particulares en estudio de $I_{B}$ se tomaron en cuenta tres parámetros espectrales, los cuales son obtenidos a partir de los espectros de pseudo-aceleración $S a$, velocidad $V$ y desplazamiento $D$, aunque pueden ser utilizados otros parámetros. En algunos casos se utilizó un solo parámetro espectral para definir $I_{B}$ (ver Caso 1, tabla 8), mientras que en otros casos se combinaron dos o tres de estos parámetros espectrales de tal manera que la medida de intensidad sísmica funcionaría como híbrida. En la tabla 8 se muestran los 30 casos particulares en estudio de $I_{B}$. 
Tabla 8. Formulación de los casos particulares en estudio de $I_{B}$

\begin{tabular}{|c|c|c|c|c|c|c|}
\hline Caso & $S\left(T_{1}\right)$ & $N_{p}$ & $R_{(T 1, T m i)}$ & Formulación & de los casos particulare & s de $I_{B}$ \\
\hline 1 & $S a$ & $S a$ & $S a$ & $I_{B(S a, S a, S a)}=S a\left(T_{1}\right)$ & {$\left[\frac{S a_{\text {avg }}\left(T_{1}, \ldots, T_{N}\right)}{\operatorname{Sa}\left(T_{1}\right)}\right]^{\alpha_{2}}$.} & {$\left[\frac{S a\left(T_{m 2}\right)}{S a\left(T_{1}\right)}\right]^{\alpha_{3}}$} \\
\hline 2 & $S a$ & $S a$ & $V$ & $I_{B(S a, S a, V)}=S a\left(T_{1}\right)$ & {$\left[\frac{S a_{\text {avg }}\left(T_{1}, \ldots, T_{N}\right)}{S a\left(T_{1}\right)}\right]^{\alpha_{2}}$} & $\cdot\left[\frac{V\left(T_{m 2}\right)}{V\left(T_{1}\right)}\right]^{\alpha_{3}}$ \\
\hline 3 & $S a$ & $S a$ & $D$ & $I_{B(S a, S a, D)}=S a\left(T_{1}\right)$ & {$\left[\frac{S a_{a v g}\left(T_{1}, \ldots, T_{N}\right)}{S a\left(T_{1}\right)}\right]^{\alpha_{2}}$} & $\cdot\left[\frac{D\left(T_{m 2}\right)}{D\left(T_{1}\right)}\right]^{\alpha_{3}}$ \\
\hline 4 & $S a$ & $V$ & $S a$ & $I_{B(S a, V, S a)}=S a\left(T_{1}\right)$ & {$\left[\frac{V_{\text {avg }}\left(T_{1}, \ldots, T_{N}\right)}{V\left(T_{1}\right)}\right]^{\alpha_{2}}$} & {$\left[\frac{S a\left(T_{m 2}\right)}{S a\left(T_{1}\right)}\right]^{\alpha_{3}}$} \\
\hline 5 & $S a$ & $V$ & $V$ & $I_{B(S a, V, V)}=S a\left(T_{1}\right)$ & {$\left[\frac{V_{a v g}\left(T_{1}, \ldots, T_{N}\right)}{V\left(T_{1}\right)}\right]^{\alpha_{2}}$} & {$\left[\frac{V\left(T_{m 2}\right)}{V\left(T_{1}\right)}\right]^{\alpha_{3}}$} \\
\hline 6 & $S a$ & $V$ & $D$ & $I_{B(S a, V, D)}=S a\left(T_{1}\right)$ & {$\left[\frac{V_{a v g}\left(T_{1}, \ldots, T_{N}\right)}{V\left(T_{1}\right)}\right]^{\alpha_{2}}$} & {$\left[\frac{D\left(T_{m 2}\right)}{D\left(T_{1}\right)}\right]^{\alpha_{3}}$} \\
\hline 7 & $S a$ & $D$ & $S a$ & $I_{B(S a, D, S a)}=S a\left(T_{1}\right)$ & {$\left[\frac{D_{\text {avg }}\left(T_{1}, \ldots, T_{N}\right)}{D\left(T_{1}\right)}\right]^{\alpha_{2}}$} & {$\left[\frac{S a\left(T_{m 2}\right)}{S a\left(T_{1}\right)}\right]^{\alpha_{3}}$} \\
\hline 8 & $S a$ & $D$ & $V$ & $I_{B(S a, D, V)}=S a\left(T_{1}\right)$ & {$\left[\frac{D_{\text {avg }}\left(T_{1}, \ldots, T_{N}\right)}{D\left(T_{1}\right)}\right]^{\alpha_{2}}$} & {$\left[\frac{V\left(T_{m 2}\right)}{V\left(T_{1}\right)}\right]^{\alpha_{3}}$} \\
\hline 9 & $S a$ & $D$ & $D$ & $I_{B(S a, D, D)}=S a\left(T_{1}\right)$ & {$\left[\frac{D_{\text {avg }}\left(T_{1}, \ldots, T_{N}\right)}{D\left(T_{1}\right)}\right]^{\alpha_{2}}$} & {$\left[\frac{D\left(T_{m 2}\right)}{D\left(T_{1}\right)}\right]^{\alpha_{3}}$} \\
\hline 10 & $V$ & $S a$ & $S a$ & $I_{B(V, S a, S a)}=V\left(T_{1}\right)$ & $\left.\frac{S a_{a v g}\left(T_{1}, \ldots, T_{N}\right)}{S a\left(T_{1}\right)}\right]^{\alpha_{2}}$ & {$\left[\frac{S a\left(T_{m 2}\right)}{S a\left(T_{1}\right)}\right]^{\alpha_{3}}$} \\
\hline 11 & $V$ & $S a$ & $V$ & $I_{B(V, S a, V)}=V\left(T_{1}\right)$. & {$\left[\frac{S a_{a v g}\left(T_{1}, \ldots, T_{N}\right)}{S a\left(T_{1}\right)}\right]^{\alpha_{2}}$} & {$\left[\frac{V\left(T_{m 2}\right)}{V\left(T_{1}\right)}\right]^{\alpha_{3}}$} \\
\hline 12 & $V$ & $S a$ & $D$ & $I_{B(V, S a, D)}=V\left(T_{1}\right)$ & {$\left[\frac{S a_{\text {avg }}\left(T_{1}, \ldots, T_{N}\right)}{S a\left(T_{1}\right)}\right]^{\alpha_{2}}$} & {$\left[\frac{D\left(T_{m 2}\right)}{D\left(T_{1}\right)}\right]^{\alpha_{3}}$} \\
\hline 13 & $V$ & $V$ & $S a$ & $I_{B(V, V, S a)}=V\left(T_{1}\right)$ & {$\left[\frac{V_{\text {avg }}\left(T_{1}, \ldots, T_{N}\right)}{V\left(T_{1}\right)}\right]^{\alpha_{2}}$} & $\left.\frac{S a\left(T_{m 2}\right)}{S a\left(T_{1}\right)}\right]^{\alpha_{3}}$ \\
\hline 14 & $V$ & $V$ & $V$ & $I_{B(V, V, V)}=V\left(T_{1}\right)$ & {$\left[\frac{V_{\text {avg }}\left(T_{1}, \ldots, T_{N}\right)}{V\left(T_{1}\right)}\right]^{\alpha_{2}}$} & $\left.\frac{V\left(T_{m 2}\right)}{V\left(T_{1}\right)}\right]^{\alpha_{3}}$ \\
\hline 15 & $V$ & $V$ & $D$ & $I_{B(V, V, D)}=V\left(T_{1}\right)$. & {$\left[\frac{V_{\text {avg }}\left(T_{1}, \ldots, T_{N}\right)}{V\left(T_{1}\right)}\right]^{\alpha_{2}}$} & $\left.\frac{D\left(T_{m 2}\right)}{D\left(T_{1}\right)}\right]^{\alpha_{3}}$ \\
\hline 16 & $V$ & $D$ & $S a$ & $I_{B(V, D, S a)}=V\left(T_{1}\right) \cdot$ & {$\left[\frac{D_{a v g}\left(T_{1}, \ldots, T_{N}\right)}{D\left(T_{1}\right)}\right]^{\alpha_{2}}$} & {$\left[\frac{S a\left(T_{m 2}\right)}{S a\left(T_{1}\right)}\right]^{\alpha_{3}}$} \\
\hline
\end{tabular}




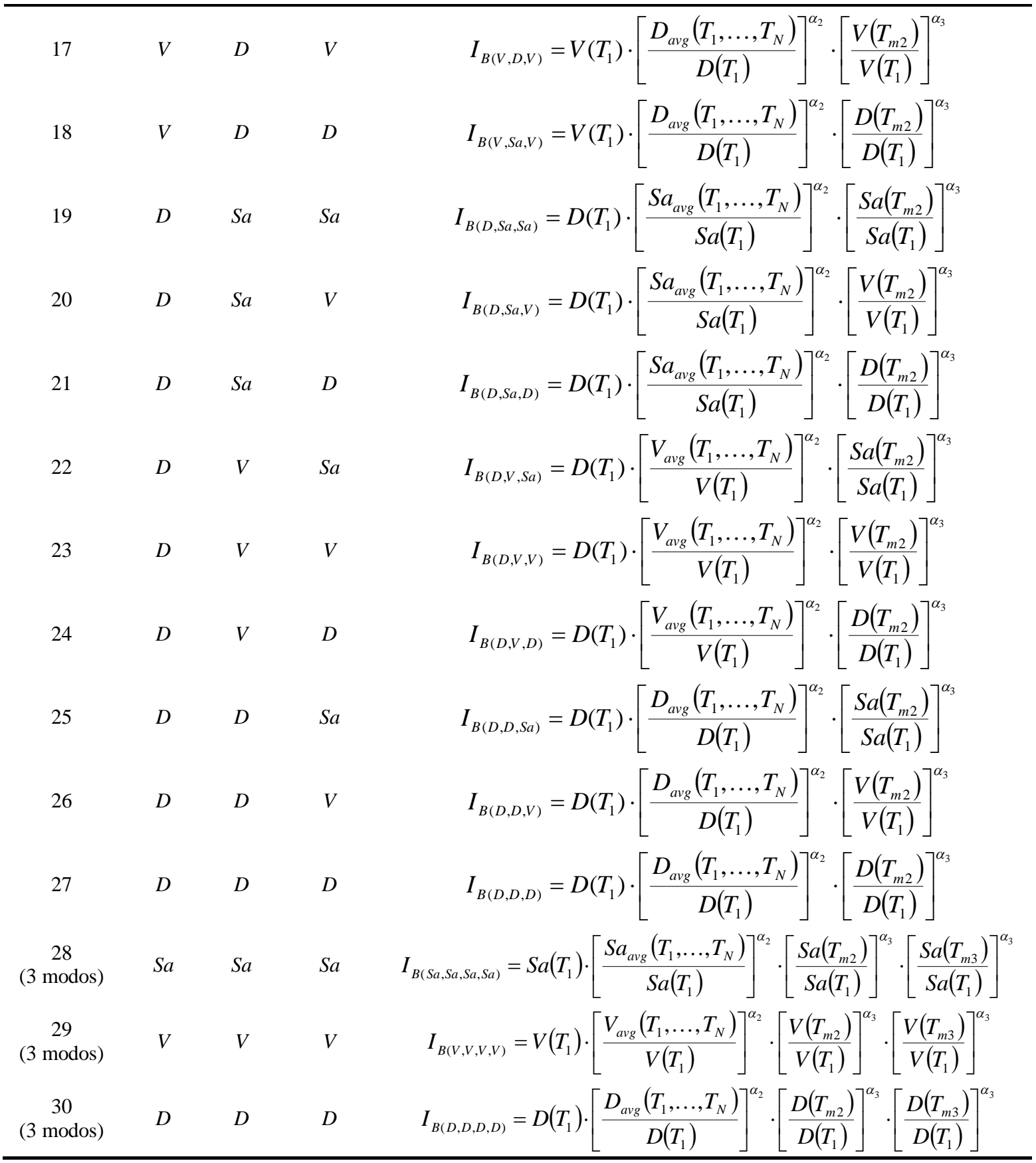

Uno de los objetivos principales de este trabajo es encontrar para cada uno de los casos particulares en estudio de $I_{B}$ los valores de $\alpha_{1}, \alpha_{2}$ y $\alpha_{3}$ para los cuales dicha medida es más eficiente; es decir, que pueda predecir con buena aproximación el comportamiento de estructuras debido a la no-linealidad y a la influencia de los modos superiores. Para ello se utiliza un análisis de regresión, como se ilustrará más adelante. Una vez que se encuentran los valores de $\alpha_{1}, \alpha_{2}$ y $\alpha_{3}$ para cada uno de los casos en estudio de $I_{B}$ se procede a comparar la desviación estándar entre los casos particulares de esta medida de intensidad sísmica para elegir los tres que presenten la mejor eficiencia y después compararlos contra la desviación estándar de $I_{N p}$ y de $S a\left(T_{1}\right)$. Con ello se pretende demostrar la eficiencia de $I_{B}$ como predictor de la 
respuesta de estructuras con comportamiento inelástico no-lineal y bajo la influencia de modos superiores, comparado con $I_{N p}$ y con $S a\left(T_{1}\right)$. Finalmente, aunque resulta de mayor complejidad estimar $I_{B}$ en comparación con otras medidas de intensidad sísmica; dado que la obtención del parámetro propuesto depende de la forma espectral, éste podría calcularse a partir de las características del sitio y la fuente por ejemplo utilizando redes neuronales artificiales (Alcantara et al., 2009, Bojórquez et al., 2012b).

\section{RESULTADOS NUMÉRICOS}

A partir de estimar la desviación estándar se puede concluir cuál de las tres medidas de intensidad sísmica presenta menor dispersión en función de la demanda, que en este caso corresponde a la distorsión máxima de entrepiso. A continuación se muestran los resultados obtenidos del estudio de eficiencia.

\section{Resultados para $I_{N p}$}

Para el caso del parámetro $I_{N p}$ se varió el exponente $\alpha$ para verificar cuál era el valor indicado dentro del intervalo entre cero y la unidad. Este exponente calibrará $I_{N p}$ hasta encontrar el valor de $\alpha$ para el cual esta medida tiene mejor relación con la demanda sísmica. Debido a que $I_{N p}$ es un caso particular de $I_{B}$ cuando se utiliza el espectro pseudo-aceleración como parámetro espectral y se suponen los exponentes $\alpha_{1}$ igual a uno y $\alpha_{3}$ igual a cero. No es necesario realizar por separado los análisis para $I_{N p}$, ya que basta con estimar la intensidad generalizada de Bojórquez para obtener el valor del exponente $\alpha$ que maximiza la eficiencia de la medida de intensidad sísmica. De hecho, no es necesario estimar $\sigma_{\ln (\gamma)}$ para $S a\left(T_{1}\right)$, ya que está medida de intensidad sísmica también es un caso particular de $I_{B}$ cuando se utiliza el espectro pseudoaceleración como parámetro espectral y considerando $\alpha_{1}$ igual a uno, $\alpha_{2}$ igual a cero y $\alpha_{3}$ igual a cero.

A razón de los resultados obtenidos por Bojórquez y Iervolino (2011) y en este presente estudio se ha concluido que el valor que minimiza la dispersión de esta medida de $I S$ es un exponente $\alpha$ igual a 0.4. Por lo tanto, $I_{N p}$ puede estimarse como se indica en la ecuación 7.

$$
I_{N p}=S a\left(T_{E}\right) \cdot N_{p}^{0.4}
$$

Los valores obtenidos de $\sigma_{\ln (\gamma)}$ al utilizar la ecuación 7 , se comparan más adelante con los resultados de las otras medidas de intensidad sísmica en estudio.

\section{Resultados para los casos de $I_{B}$}

Para el análisis de los casos en estudio de $I_{B}$ se tomó en cuenta únicamente los parámetros para el segundo y tercer modo de vibrar (ver tabla 8). Note que la incorporación del segundo modo de vibrar para predecir la respuesta estructural es suficiente para la mayoría de las edificaciones; sin embargo, en este trabajo se incluyó el tercer modo vibrar con la finalidad demostrar que no es necesario su empleo ya que no contribuye de manera significativa en la predicción de la respuesta estructural al menos para las estructuras analizadas. Durante el análisis se variaron los exponentes, $\alpha_{1}, \alpha_{2}$ y $\alpha_{3}$, a partir del cambio de los valores de estos factores se construyeron las gráficas presentadas de la figura 8 a la figura 13. Este conjunto de figuras corresponden a $I_{B(S a, S a, S a)}$ (Caso 1), en las cuales se muestran en los ejes horizontales las variaciones de los exponente $\alpha_{2}$ y $\alpha_{3}$, y en el eje vertical se gráfica la desviación estándar del logaritmo natural de la distorsión máxima de entrepiso. En estas figuras también se muestra el cambio del parámetro $T_{N}$ (elongación del periodo de la estructura debido al comportamiento no lineal). Recordando que la medida de intensidad sísmica $I_{B}$ involucra el factor $N_{p}$, este se calcula mediante la ecuación 4 . Se observa que el periodo $T_{N}$ tiene gran importancia debido a que involucra directamente información sobre la forma 
del espectro de respuesta. Cabe mencionar que en el presente trabajo se utilizaron para la determinación de $N_{p}$ varios puntos intermedios considerando ordenadas espectrales a cada 0.1 segundos de periodo.

Las figuras 8-13 muestran algunos resultados obtenidos para la mayoría de los registros sísmicos seleccionados, así como de dos clasificaciones adicionales donde se calculó la desviación estándar para todos los tipos de suelo en forma simultánea, esta clasificación se le llama TOTAL, pero se hizo sin involucrar el tipo de suelo MB. Después, se calcula la desviación estándar de todos los tipos pero ahora incluyendo el suelo MB, llamado TOTMB. Esto se da un total de 6 tipos de suelos, 2 tipos combinados para 6 marcos estructurales diferentes. Dichos resultados se obtuvieron con la finalidad de encontrar los valores de $\alpha_{1}, \alpha_{2}$ y $\alpha_{3}$ que presentan las menores desviaciones estándar, y por lo tanto que reducen la incertidumbre asociada en la predicción de la respuesta estructural de edificaciones sometidas a sismos de diversas características.

En primer lugar, en la figura 8 se ilustran los resultados para el marco de 4 niveles y los registros sísmicos de suelo tipo A para $I_{B(S a, S a, S a)}$. Se observa para las cuatro superficies que hay una región donde se encuentran los valores más bajos de la desviación estándar. Lo más destacable es que para los movimientos obtenidos de suelo duro (tipo A) se observa un valle en la figura 8 para cualquier valor de $T_{N}$; en particular los mínimos valores se presentan cuando $T_{N}=2 T_{1}$ como lo sugieren Bojórquez y Iervolino (2011). En el caso de los marcos de 6, 8, 10, 14 y 18 niveles (ver figuras 9 a 13) se puede observar como las superficies definen claramente un valle en el cual se encuentra la desviación estándar mínima, esto se debe a que el segundo modo de vibrar empieza a tener un efecto significativo sobre la estructura. Esta región se ve delimitada por un par de intervalos, uno para $\alpha_{2}$ y el otro para $\alpha_{3}$. En el intervalo para $\alpha_{2}$ se observa que la región de interés está entre 0.2 a 0.4 y para el otro exponente varía desde 0.1 a 0.3 , para la gran mayoría de las superficies presentadas. Lo anterior también se observa para el marco de 4 niveles analizado con los registros de suelo tipo A.

Bojórquez y Iervolino (2011) concluyeron que $\alpha=0.4$ es el valor que permite a $I_{N p}$ tener la mayor eficiencia al usar $I_{N p}$ como medida de $I S$. Esto puede observarse en las figuras 8 a 13 que indican que $\alpha_{2}=0.4$ es un valor razonable cuando $\alpha_{3}=0$. En este estudio también se adoptó este mismo valor para el uso de $I_{N p}$ y para $I_{B(S a, S a, S a)}$ debido a que los resultados obtenidos en este estudio concuerdan con los obtenidos por Bojórquez y Iervolino (2011). Para $\alpha_{3}$ se propone un valor de 0.2, en función de lo observado en las superficies, ya que un valor de $\alpha_{3}=0.2$ forma parte del valle de la superficie, donde la desviación estándar se reduce en gran medida. De acuerdo a los resultados obtenidos en el presente estudio se puede concluir que el conjunto de valores $\alpha_{1}, \alpha_{2}$ y $\alpha_{3}$ que reducen la desviación estándar para $I_{B(S a, S a, S a)}$ son $1,0.4$ y 0.2 respectivamente. Utilizando el espectro de pseudo-aceleración como parámetro espectral y sustituyendo los valores de $\alpha_{1}, \alpha_{2}$ y $\alpha_{3}$ en la ecuación 3 se obtiene $I_{B(S a, S a, S a)}$ y se puede reescribir la expresión como lo indica la ecuación 8 . Note que la ecuación 8 tiene incluidos los subíndices $S a$ en $I_{B}$, esta nomenclatura se utilizó debido a que todos los parámetros involucrados dependen de la pseudo-aceleración.

$$
I_{B(S a, S a, S a)}=S a\left(T_{1}\right) \cdot N_{p}^{0.4} \cdot R_{T 1, T 2}^{0.2}
$$

Es importante mencionar que para todas las figuras cuando $\alpha_{2}$ y $\alpha_{3}$ son iguales a cero se obtiene $S a\left(T_{1}\right)$. Para este caso, se observa que $\sigma_{\ln (\gamma)}$ es mucho mayor para $S a\left(T_{1}\right)$ que cuando $\alpha_{2}$ y $\alpha_{3}$ son diferentes de cero. Por lo tanto, se puede concluir que las medidas de intensidad sísmica $I_{N p}$ e $I_{B(S a, S a, S a)}$, están más relacionadas con la respuesta estructural. Además, note que debido a los que los parámetros $N_{p}$ y $R_{T 1, T 2}$ son adimensionales, la intensidad $I_{B}$ tendrá las unidades del parámetro utilizado, donde en la ecuación 8 corresponde a las unidades de pseudo-aceleración, esto es gracias a que $\alpha_{l}$ es igual a la unidad. 

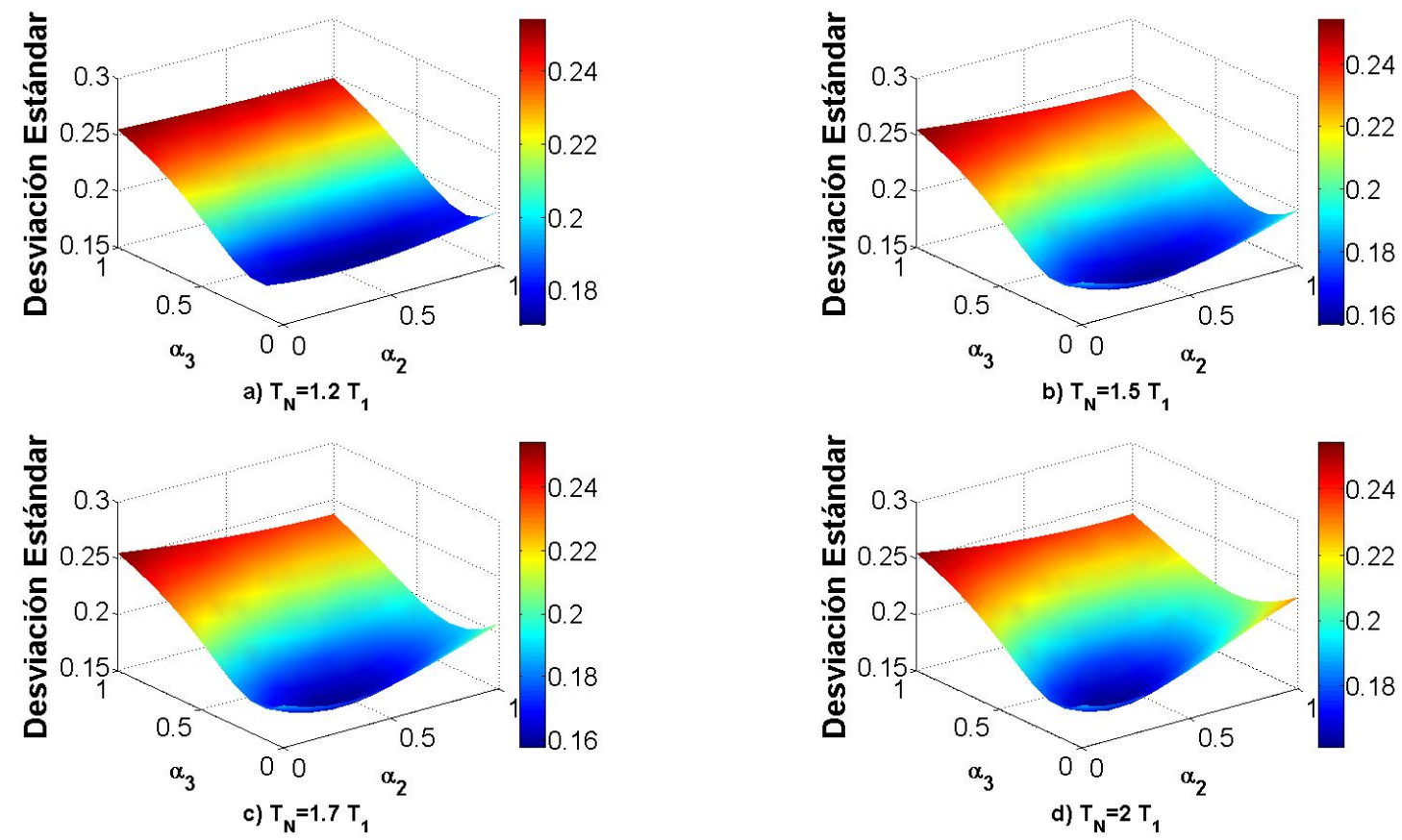

Figura 8. $\sigma_{\ln (\gamma)}$ para marco de 4 niveles sometido a movimientos sísmicos de suelo tipo A $\left(I_{B(S a, S a, S a)}\right)$

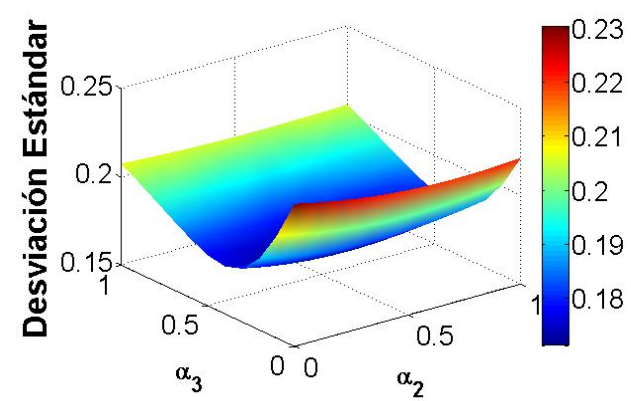

a) $\mathrm{T}_{\mathrm{N}}=1.2 \mathrm{~T}_{1}$

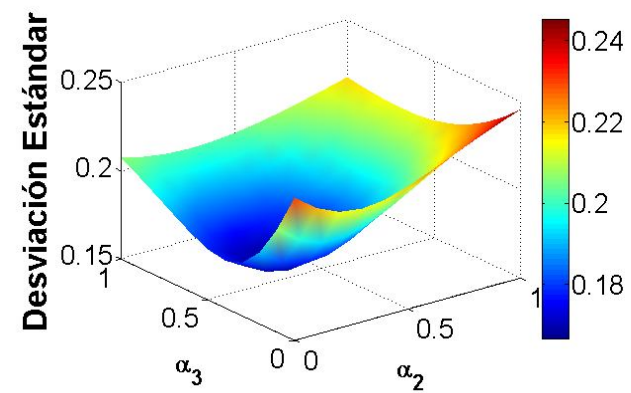

c) $\mathrm{T}=1.7 \mathrm{~T}$

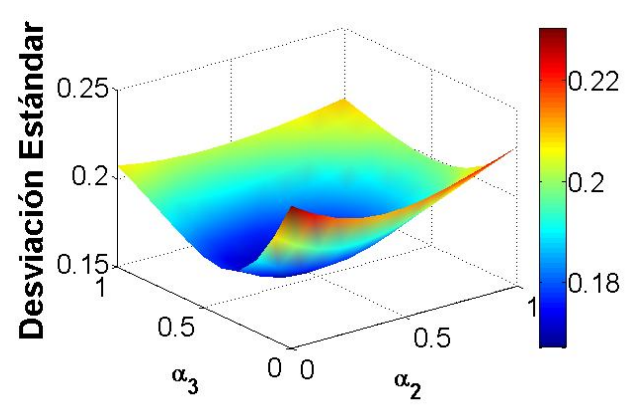

b) $\mathrm{T}_{\mathrm{N}}=1.5 \mathrm{~T}_{1}$

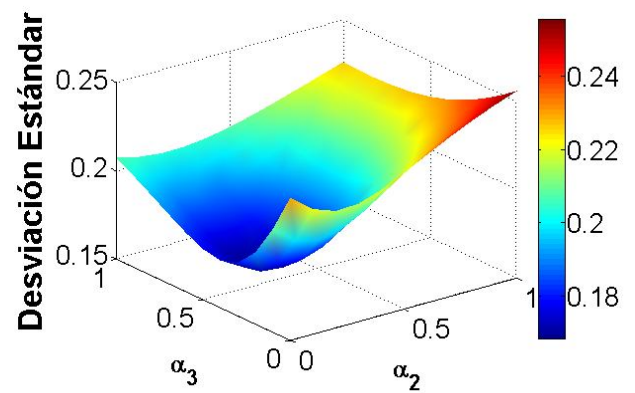

d) $\mathrm{T}=2 \mathrm{~T}$

Figura 9. $\sigma_{\ln (\gamma)}$ para marco de 6 niveles sometido a movimientos sísmicos de suelo tipo $\mathrm{C}\left(I_{B(S a, S a, S a)}\right)$ 

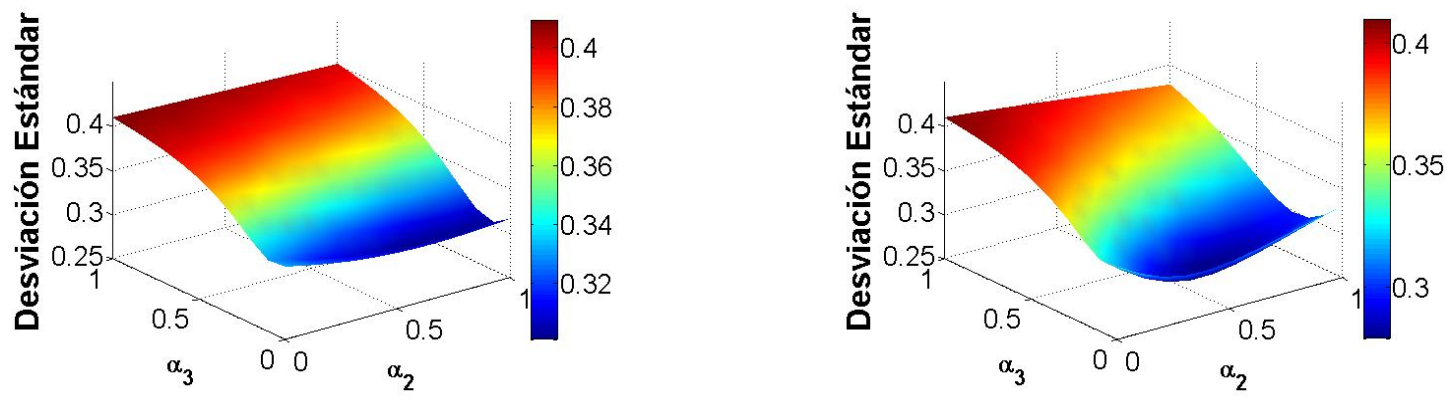

a) $T_{N}=1.2 T_{1}$

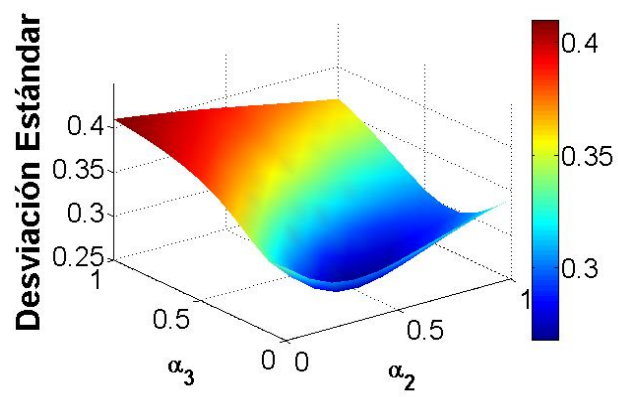

c) $\mathrm{T}_{\mathrm{N}}=1.7 \mathrm{~T}_{1}$

b) $\mathrm{T}_{\mathrm{N}}=1.5 \mathrm{~T}_{1}$

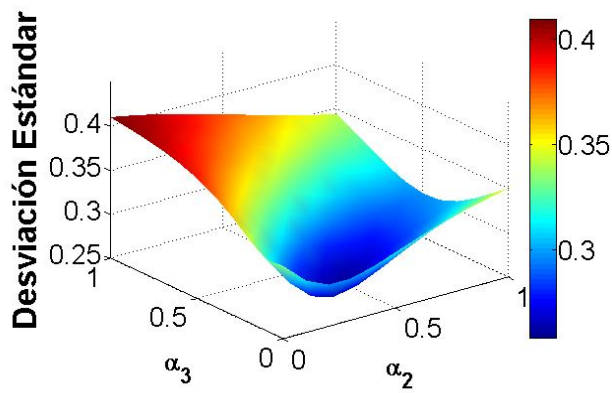

d) $T_{N}=2 T_{1}$

Figura 10. $\sigma_{\ln (\gamma)}$ para marco de 8 niveles sometido a movimientos sísmicos de suelo tipo TOTMB $\left(I_{B(S a, S a, S a)}\right)$
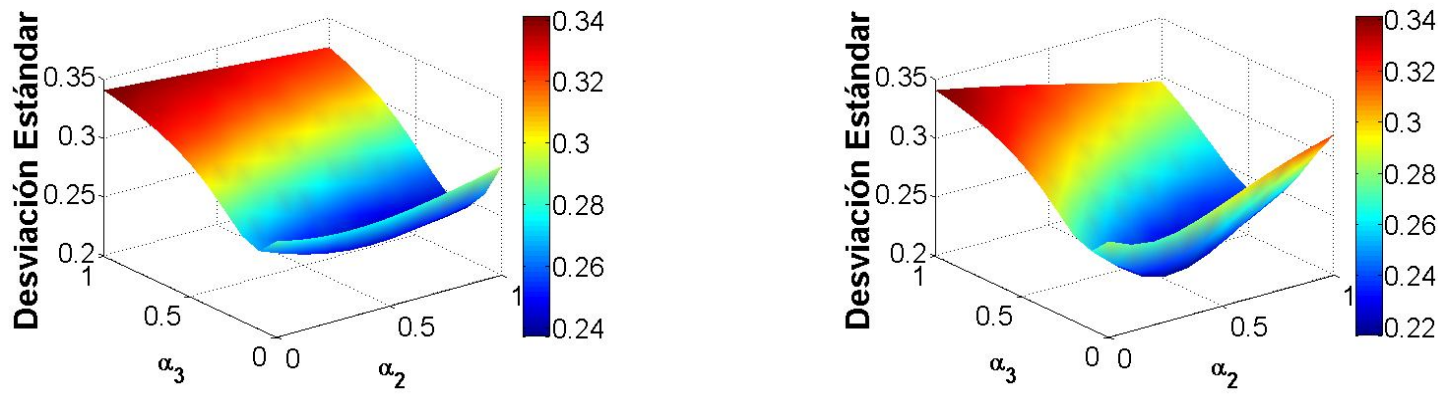

a) $\mathrm{T}_{\mathrm{N}}=1.2 \mathrm{~T}_{1}$

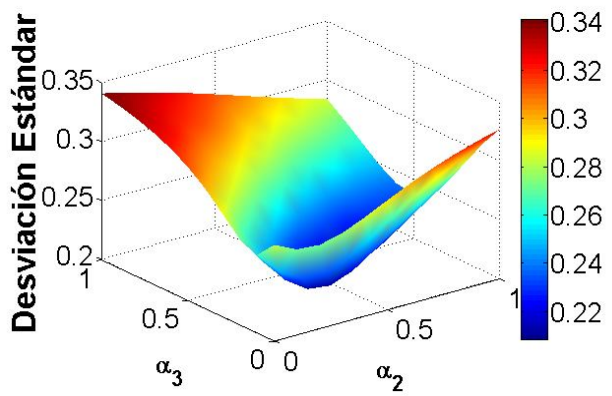

c) $\mathrm{T}_{\mathrm{N}}=1.7 \mathrm{~T}_{1}$

b) $\mathrm{T}_{\mathrm{N}}=1.5 \mathrm{~T}_{1}$

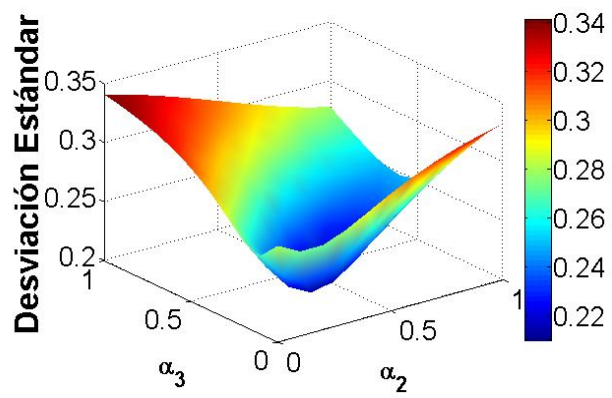

d) $T_{N}=2 T_{1}$

Figura 11. $\sigma_{\ln (\gamma)}$ para marco de 10 niveles sometido a movimientos sísmicos de suelo tipo TOTMB

$\left(I_{B(S a, S a, S a)}\right)$ 

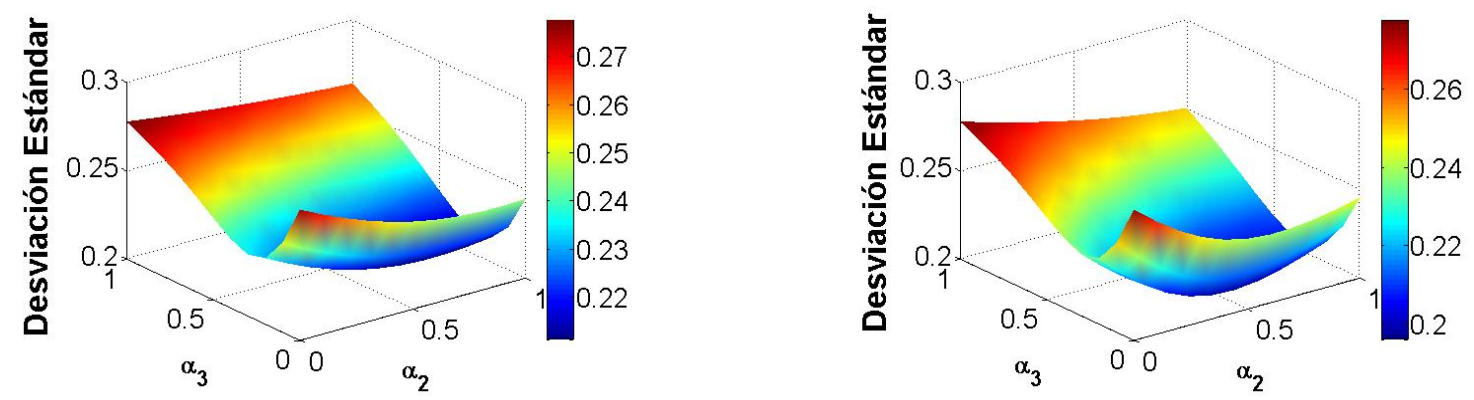

a) $T_{N}=1.2 T_{1}$

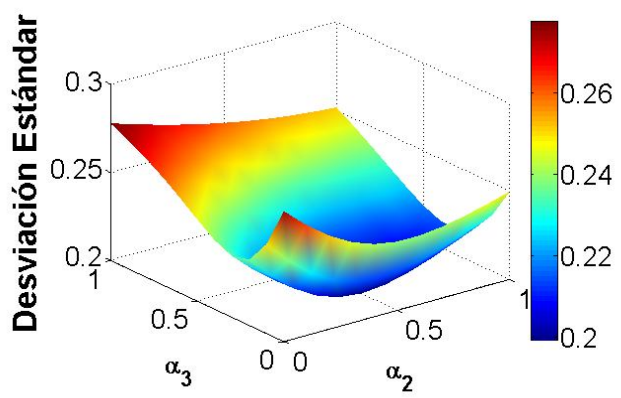

c) $\mathrm{T}_{\mathrm{N}}=1.7 \mathrm{~T}_{1}$

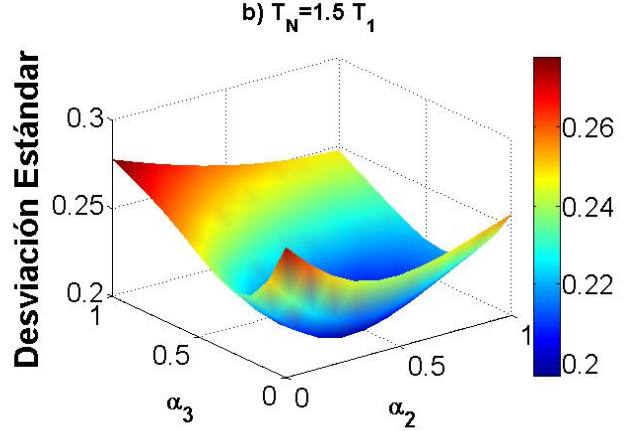

d) $T_{N}=2 T_{1}$

Figura 12. $\sigma_{\ln (\gamma)}$ para marco de 14 niveles sometido a movimientos sísmicos de suelo tipo $\mathrm{D}\left(I_{B(S a, S a, S a)}\right)$

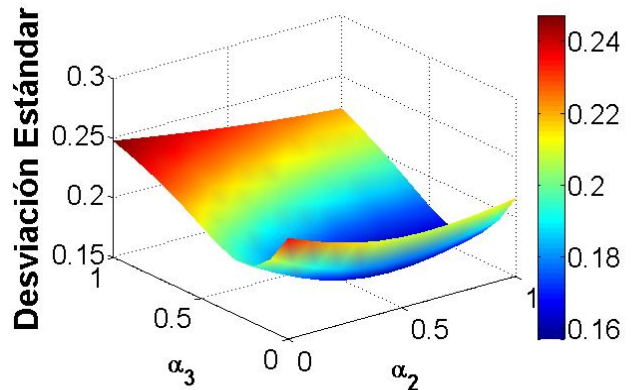

a) $T_{N}=1.2 T_{1}$

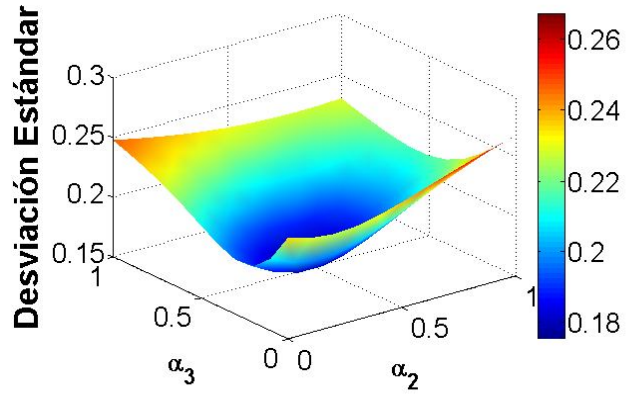

c) $\mathrm{T}_{\mathrm{N}}=1.7 \mathrm{~T}_{1}$

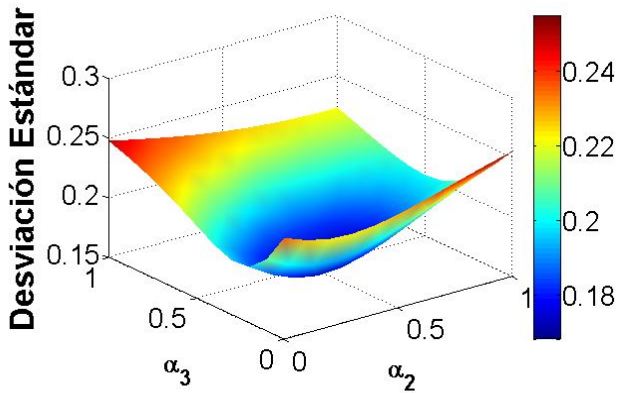

b) $\mathrm{T}_{\mathrm{N}}=1.5 \mathrm{~T}_{1}$

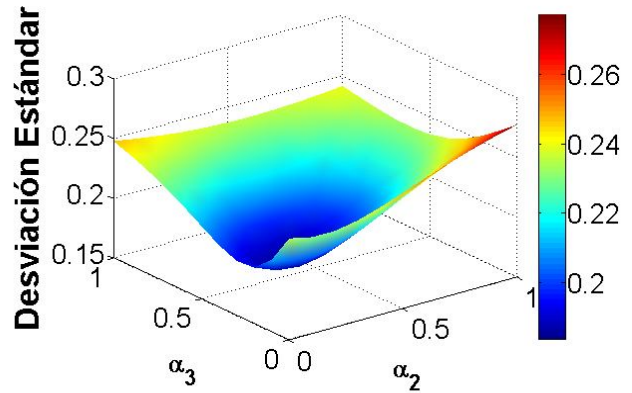

d) $\mathrm{T}_{\mathrm{N}}=2 \mathrm{~T}_{1}$

Figura 13. $\sigma_{\ln (\gamma)}$ para marco de 18 niveles sometido a movimientos sísmicos de suelo tipo $\mathrm{E}\left(I_{B(S a, S a, S a)}\right)$ 
Para obtener los resultados de los demás casos en estudio de $I_{B}$ se realizó el procedimiento mostrado anteriormente y con ello fue posible obtener los valores $\alpha_{1}, \alpha_{2}$ y $\alpha_{3}$ que calibran el resto de los casos en estudio de $I_{B}$. En la tabla 9 se muestran los tres casos que presentaron los mejores resultados (mayor eficiencia en la predicción de la distorsión máxima de entrepiso) con sus respectivos parámetros $\alpha_{1}, \alpha_{2}$ y $\alpha_{3}$ definidos. Se observa, que los exponentes son similares para los tres casos más eficientes. De hecho, estos exponentes resultaron los más adecuados en general para todos los casos analizados de la intensidad generalizada de Bojórquez.

Tabla 9. Casos en estudio que presentan la mayor eficiencia para $I_{B}$

\begin{tabular}{ccccc}
\hline Caso & $S\left(T_{1}\right)$ & $N_{p}$ & $R_{(T 1, T m i)}$ & Formulación de los casos particulares de $I_{\boldsymbol{B}}$ \\
\hline 1 & $S a$ & $S a$ & $S a$ & $I_{B(S a, S a, S a)}=S a\left(T_{1}\right) \cdot\left[\frac{S a_{a v g}\left(T_{1}, \ldots, T_{N}\right)}{S a\left(T_{1}\right)}\right]^{\alpha_{2}} \cdot\left[\frac{S a\left(T_{m 2}\right)}{S a\left(T_{1}\right)}\right]^{\alpha_{3}}$ \\
4 & $S a$ & $V$ & $S a$ & $I_{B(S a, V, S a)}=S a\left(T_{1}\right) \cdot\left[\frac{V_{\text {avg }}\left(T_{1}, \ldots, T_{N}\right)}{V\left(T_{1}\right)}\right]^{\alpha_{2}} \cdot\left[\frac{S a\left(T_{m 2}\right)}{S a\left(T_{1}\right)}\right]^{\alpha_{3}}$ \\
13 & $V$ & $V$ & $S a$ & $I_{B(V, V, S a)}=V\left(T_{1}\right) \cdot\left[\frac{V_{\text {avg }}\left(T_{1}, \ldots, T_{N}\right)}{V\left(T_{1}\right)}\right]^{\alpha_{2}} \cdot\left[\frac{S a\left(T_{m 2}\right)}{S a\left(T_{1}\right)}\right]^{\alpha_{3}}$ \\
\hline
\end{tabular}

\section{Comparación de resultados}

Una vez definidos los valores de $\alpha_{1}, \alpha_{2}$ y $\alpha_{3}$ para los casos en estudio de $I_{B}$, se procedió a efectuar una comparación entre los resultados de $S a\left(T_{1}\right), I_{N p}$ y los casos particulares de $I_{B}$ considerados en el presente estudio. En la figura 14 se muestra la desviación estándar del logaritmo natural de la distorsión máxima de entrepiso para todos los casos en estudio de $I_{B}$ para el marco de 10 niveles y todos los tipos de suelo, se observa como varia la desviación estándar para cada uno de los casos pero debido al gran número de casos en estudio se complica identificar un caso en específico, por este motivo se tomó la decisión de mostrar en las siguientes graficas únicamente los tres casos que presentaron los mejores resultados. La figura 15 muestra las desviación estándar del logaritmo natural de la distorsión máxima de entrepiso utilizando $S a\left(T_{1}\right), I_{N p}$ y los mejores casos de $I_{B}$ para el marco de 4 niveles. Se observa que $I_{N p}$ y los casos de $I_{B}$ presentan menor desviación estándar que $S a\left(T_{1}\right)$ en la mayoría de los tipos de suelo, debido a que $S a\left(T_{1}\right)$ no es buen predictor de la respuesta estructural cuando la estructura exhibe presencia de comportamiento no-lineal, de la misma manera se observa que los casos de $I_{B}$ presentan en la mayoría de los tipos de suelo menor desviación estándar que $I_{N p}$, la figura ilustra que la diferencia en los valores de la desviación estándar entre las medidas de intensidad sísmica comparadas no es muy significativa, esto se debe a que el marco de 4 niveles se encuentra dominado principalmente por su primer modo de vibrar, y el segundo modo de vibrar no afecta de manera considerable a la estructura.

En las figuras 16 a 20 se muestran las desviaciones estándar para $S a\left(T_{l}\right), I_{N p}$ y los casos de $I_{B}$ de la tabla 9 para los marcos de 6, 8, 10, 14 y 18 niveles, en los cuales se espera mayor influencia de los modos superiores de vibración. Se observa como los casos de $I_{B}$ presenta en la gran mayoría de los tipos de suelo menor desviación estándar en la respuesta sísmica en comparación con $S a\left(T_{l}\right)$ e $I_{N p}$, lo cual permite confirmar que los casos de $I_{B}$ son buenos predictores de la respuesta estructural cuando la estructura exhibe presencia de comportamiento no lineal y efecto de los modos superiores de vibración. Para presentar mayor evidencia de la influencia de los modos superiores en la respuesta estructural de algunos edificios analizados, más adelante se grafica la distribución de distorsiones máximas de entrepiso en la altura. 


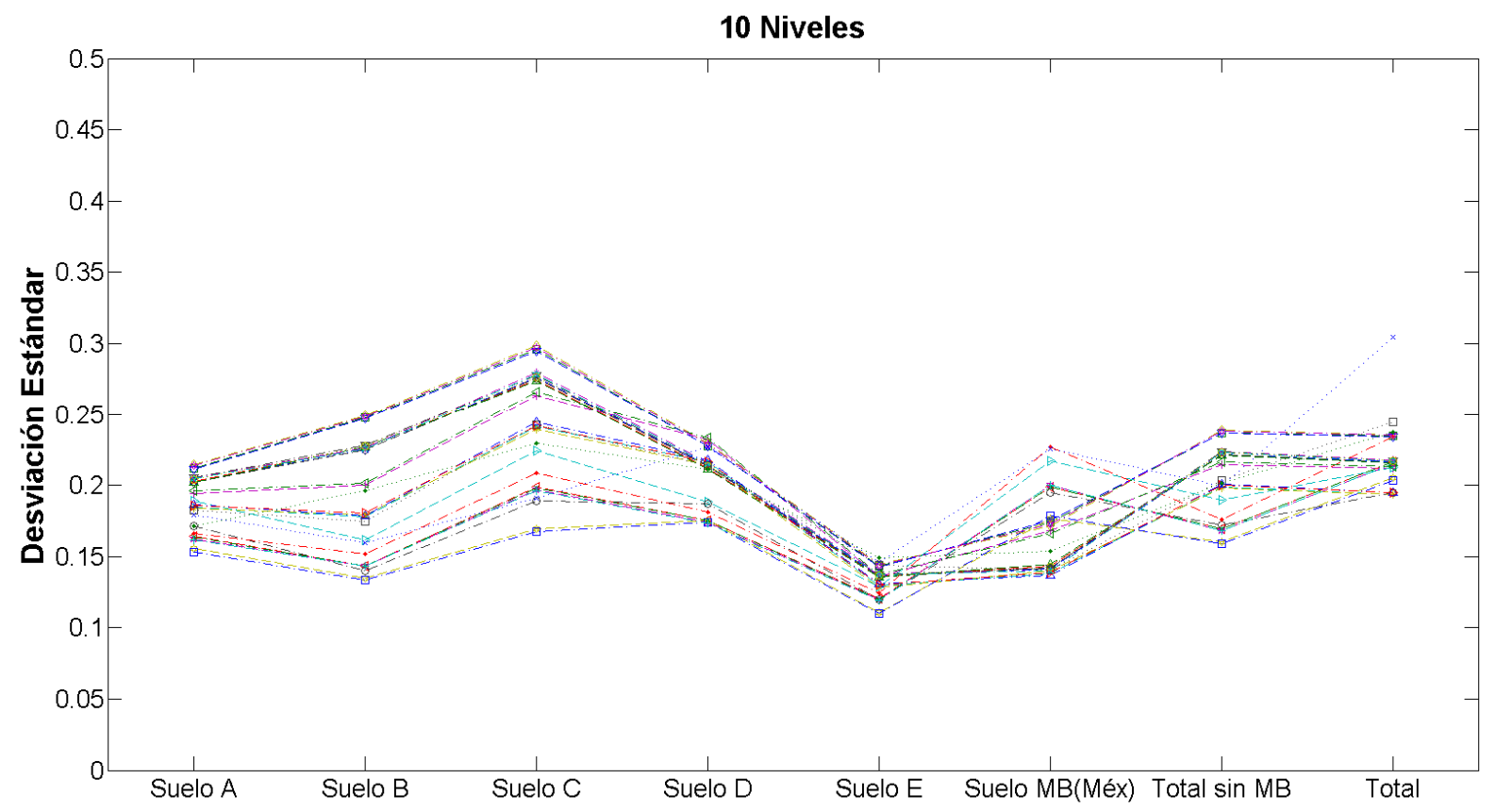

Figura 14. Comparación de $\sigma_{\ln (\gamma)}$ para el marco de 10 niveles, todos los tipos de suelo y para todos los casos en estudio de $I_{B}$

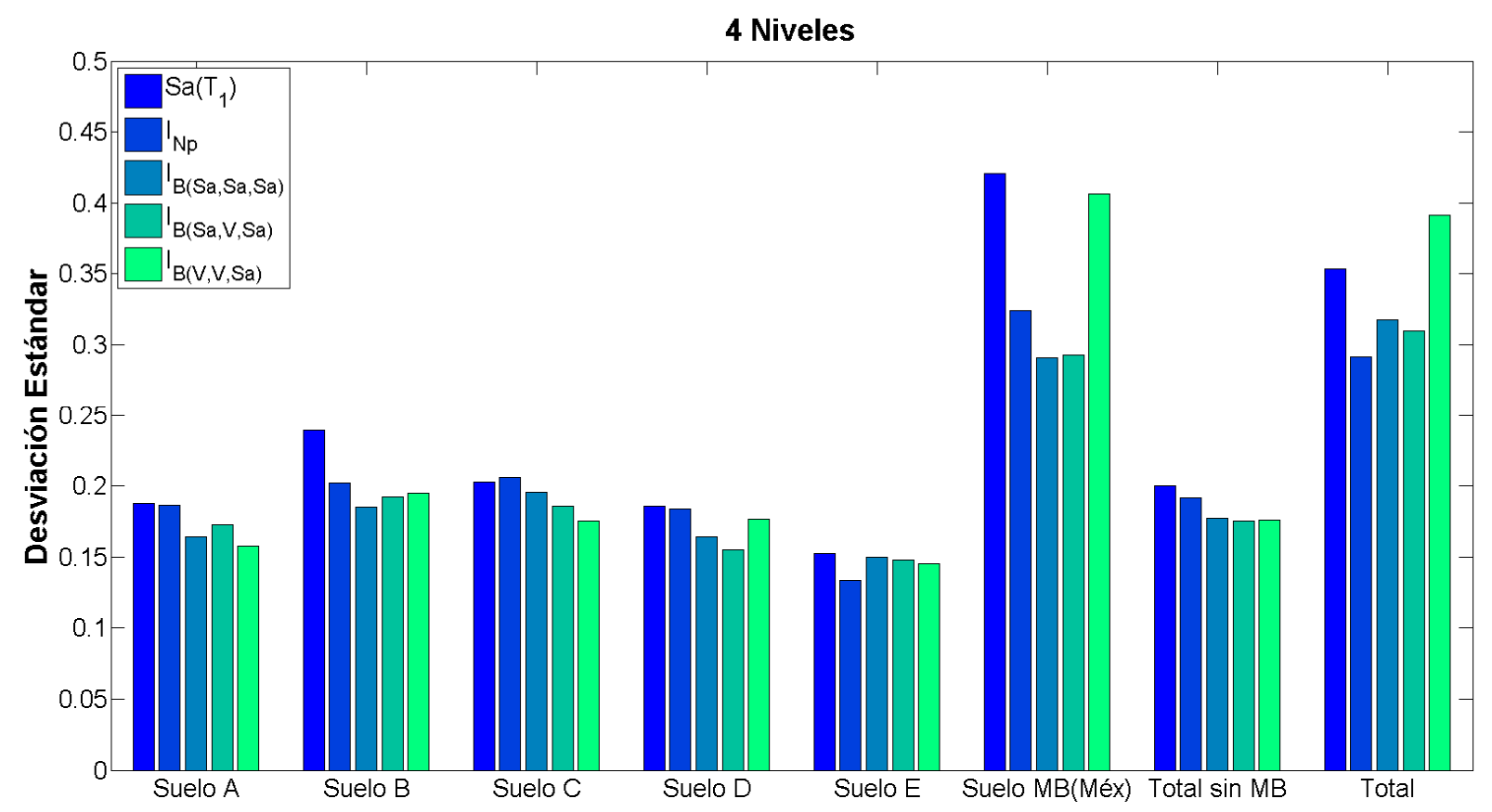

Figura 15. Comparación de $\sigma_{\ln (\gamma)}$ para el marco de 4 niveles y todos los tipos de suelo 


\section{Niveles}

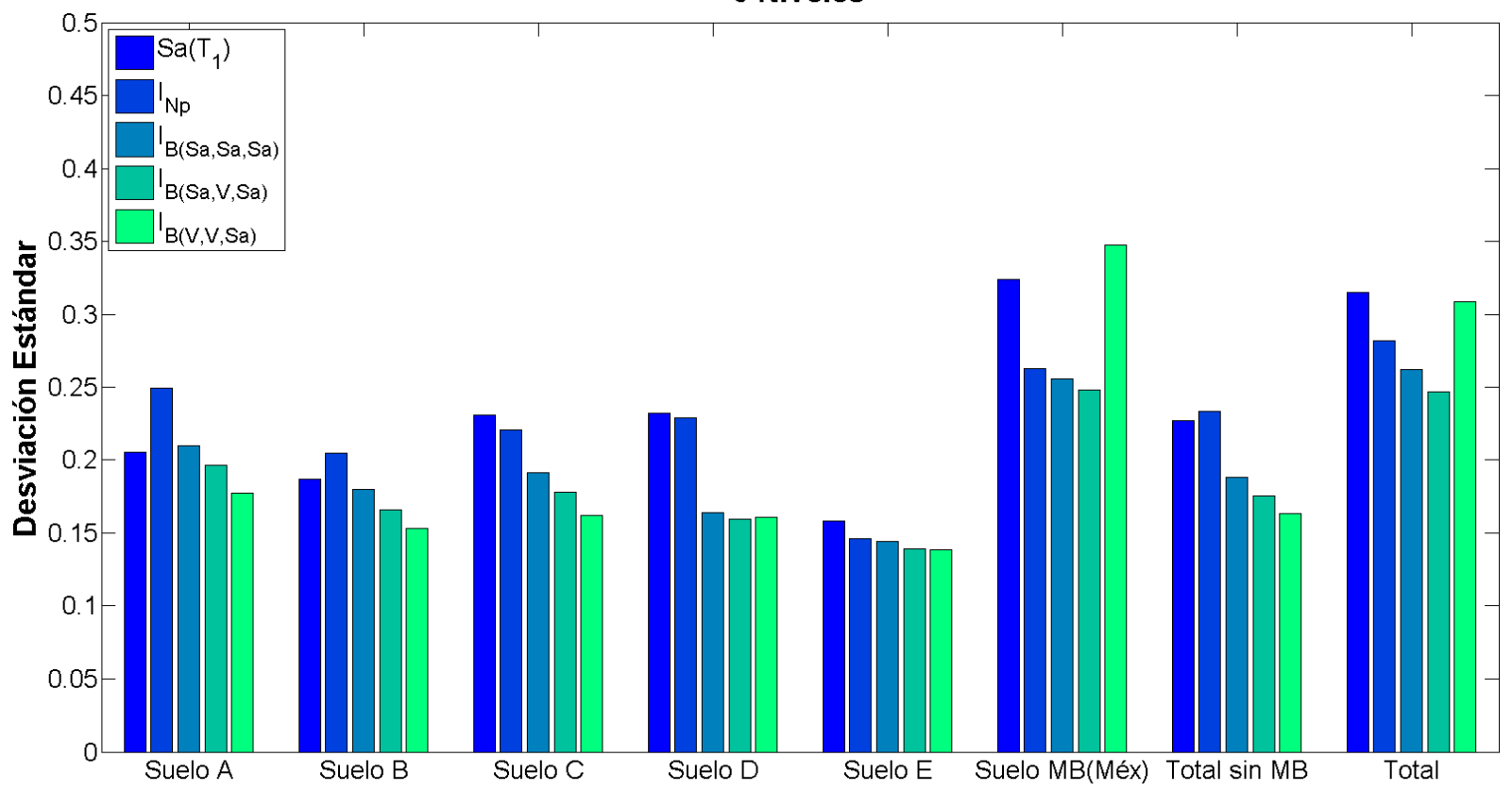

Figura 16. Comparación de $\sigma_{\ln (\gamma)}$ para el marco de 6 niveles y todos los tipos de suelo

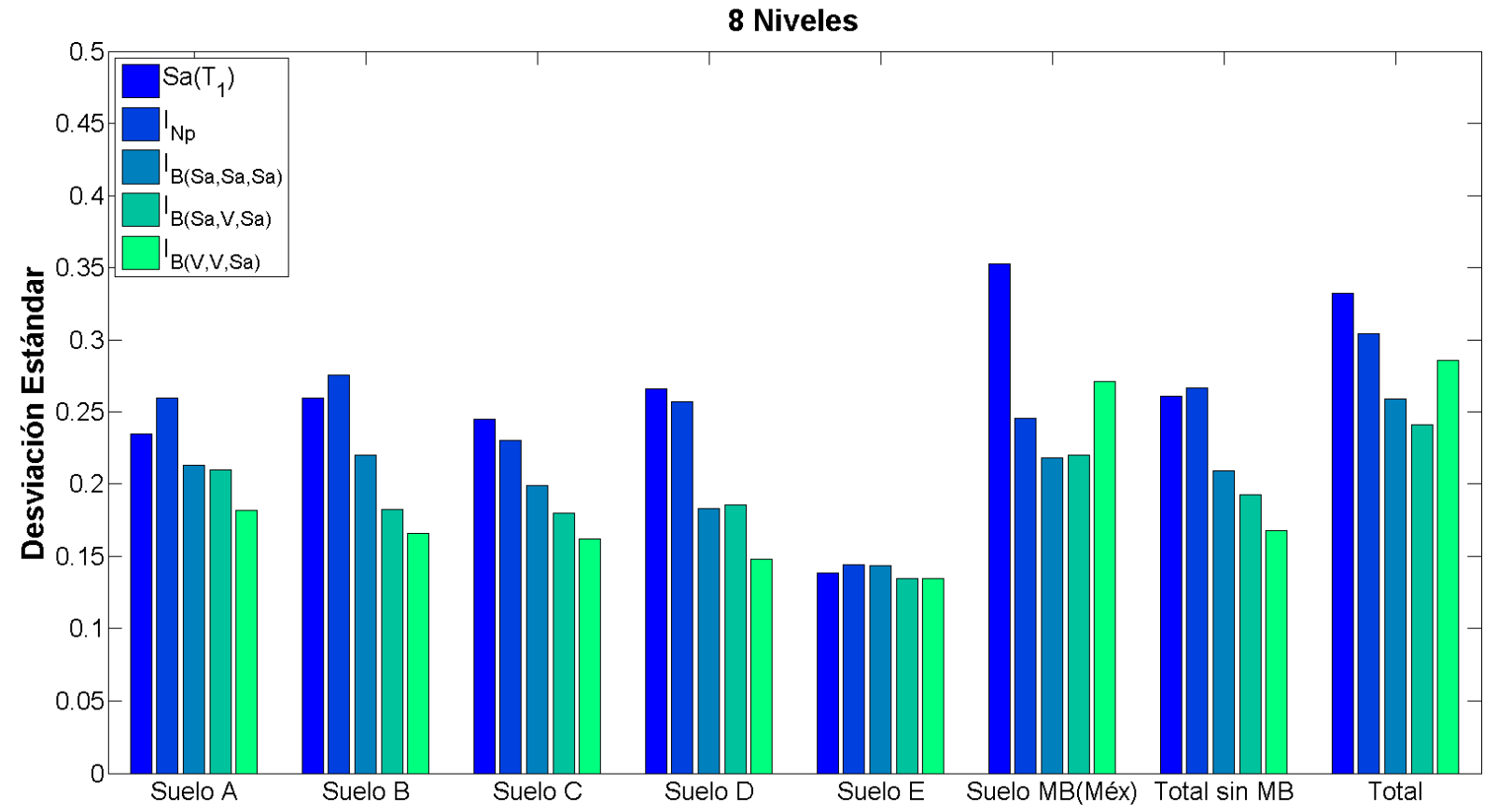

Figura 17. Comparación de $\sigma_{\ln (\gamma)}$ para el marco de 8 niveles y todos los tipos de suelo 


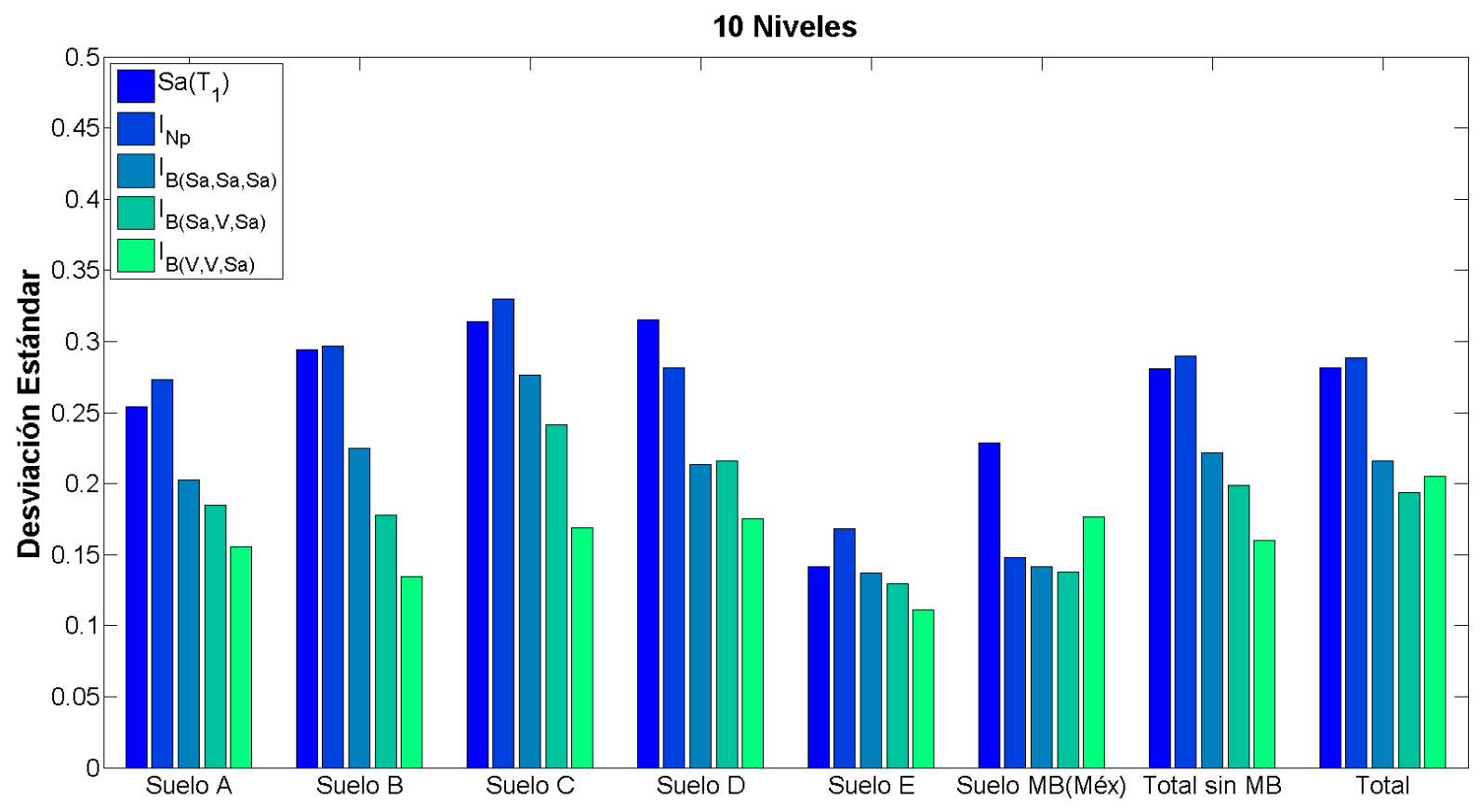

Figura 18. Comparación de $\sigma_{\ln (\gamma)}$ para el marco de 10 niveles y todos los tipos de suelo

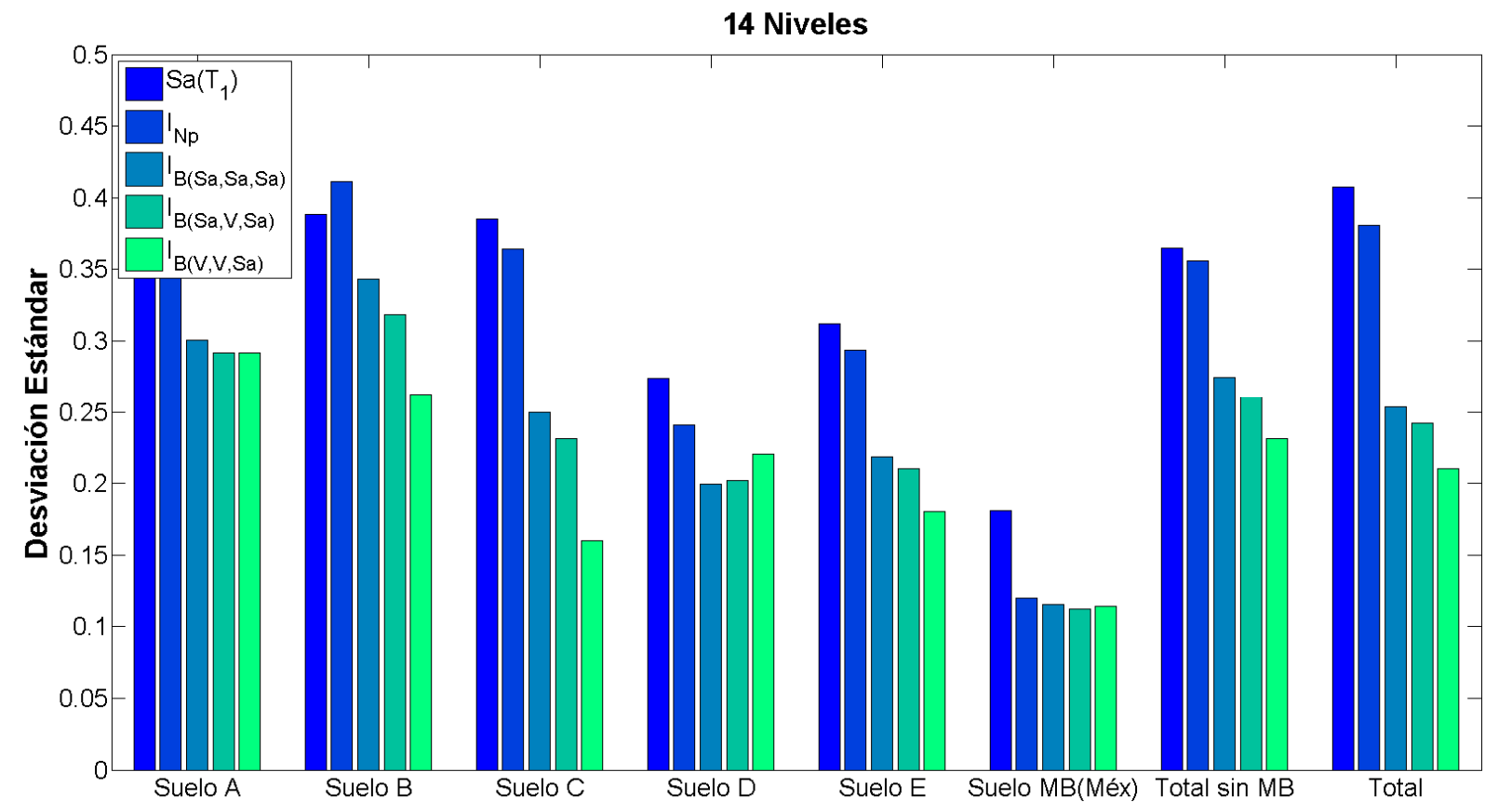

Figura 19. Comparación de $\sigma_{\ln (\gamma)}$ para el marco de 14 niveles y todos los tipos de suelo 


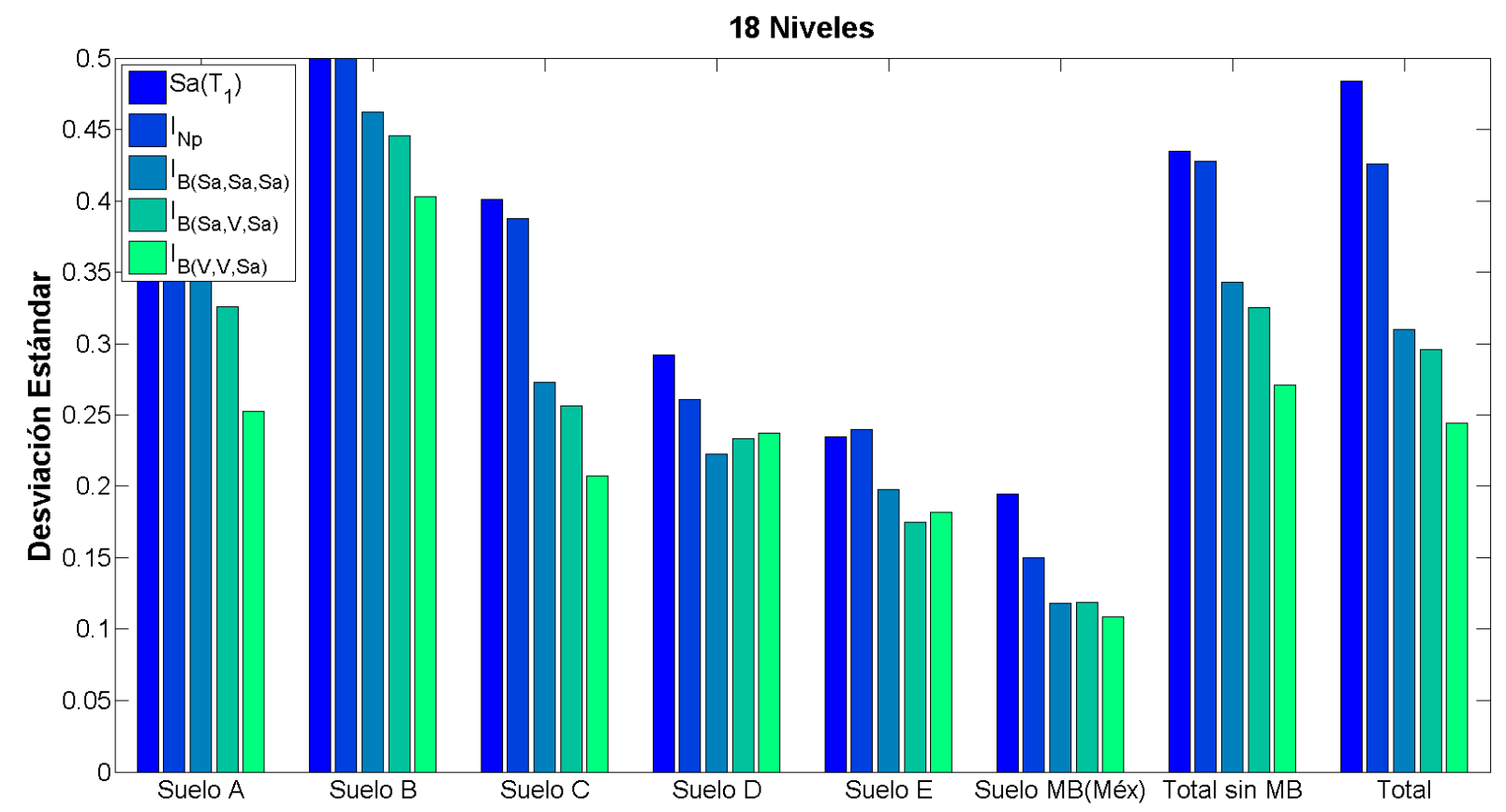

Figura 20. Comparación de $\sigma_{\ln (\gamma)}$ para el marco de 18 niveles y todos los tipos de suelo

\section{Influencia de los modos superiores}

Como se menciono anteriormente la medida de intensidad sísmica $I_{B}$ es capaz de considerar el efecto de los modos superiores de vibrar sobre sistemas estructurales; sin embargo, no se había presentado evidencia de que hubiera un efecto significativo por ejemplo del segundo modo en las estructuras utilizadas en este trabajo. Con la finalidad de demostrar el efecto del segundo modo de vibración o de modos superiores, se grafica el perfil de distorsiones de entrepiso de algunas estructuras con la altura. La figura 21 muestra el perfil de distorsiones de entrepiso para los marcos de 8, 10, 14 y 18 niveles que son las edificaciones más altas para valores de distorsión máxima de entrepiso superiores a 0.012 . Note que en estas edificaciones se espera mayor influencia de los modos superiores. En la figura se observa que para todos los marcos mostrados se presentan las distorsiones máximas de entrepiso en los niveles superiores, lo cual demuestra que estas edificaciones se ven influenciadas por modos superiores de vibrar en su respuesta sísmica. 


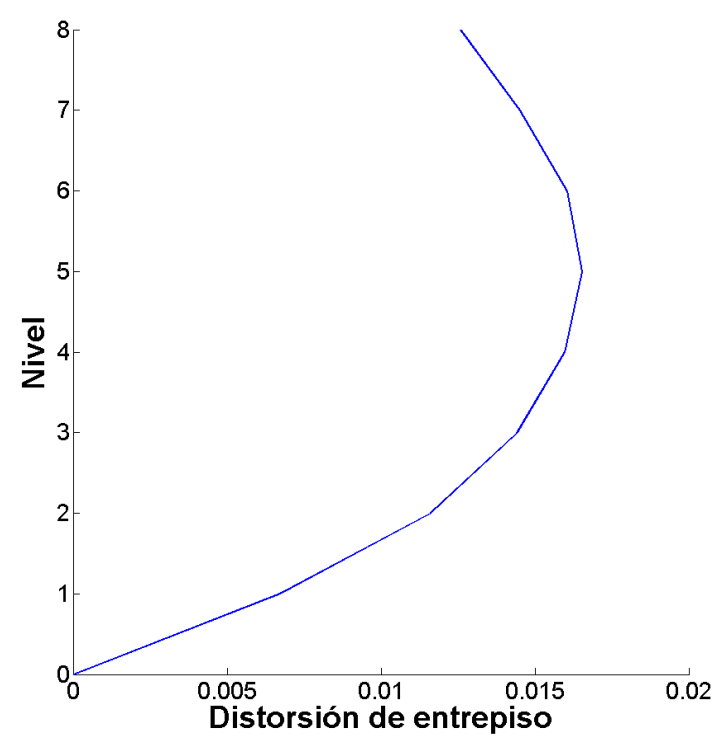

a)

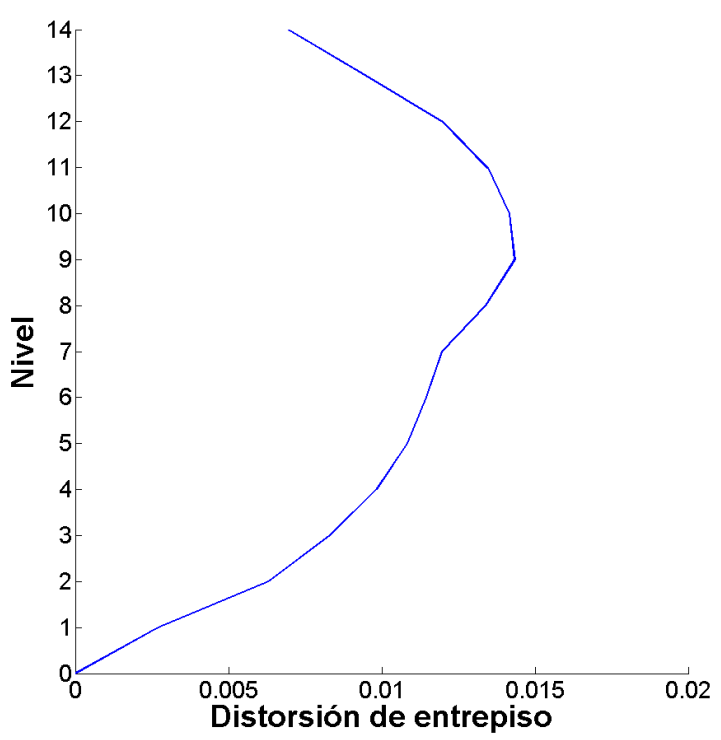

c)

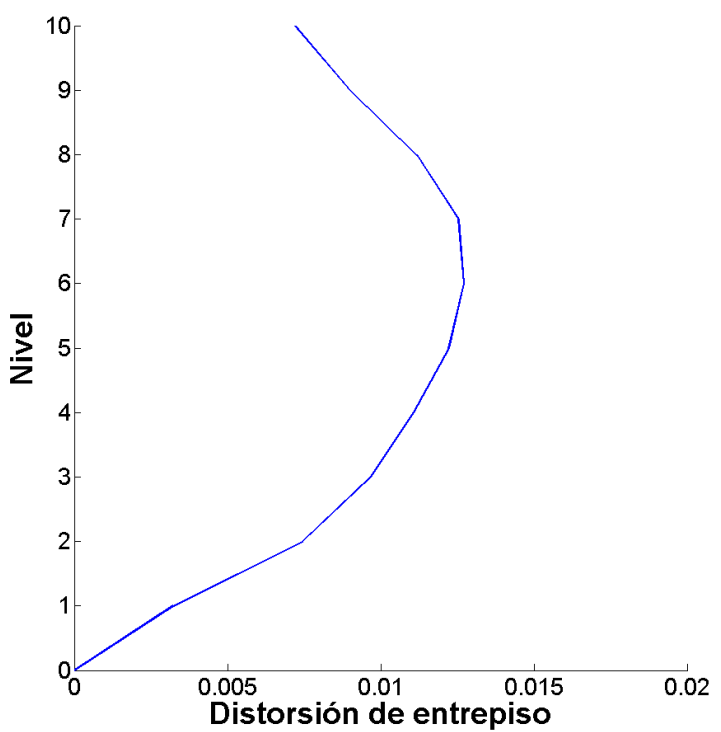

b)

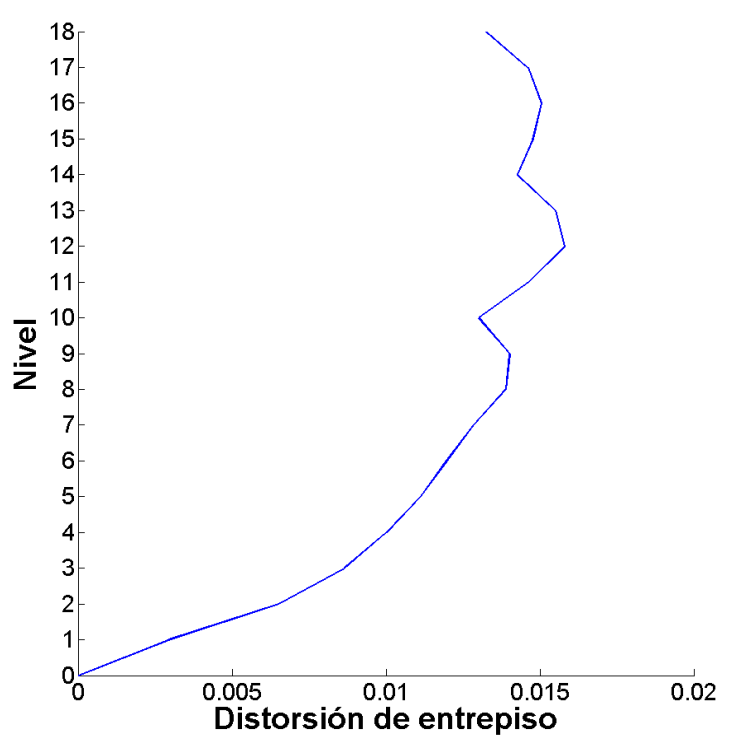

d)

Figura 21. Perfil de distorsiones de entrepiso debidas a los registros sísmicos del suelo tipo B para los marcos de: a) 8 niveles, b) 10 niveles, c) 14 niveles y d) 18 niveles

\section{Análisis dinámico incremental}

Para representar la dispersión de la demanda sísmica dada una medida de intensidad, se obtuvieron las figuras 22 a 24 que representan análisis dinámico incremental en términos de $S a$ (figura 22) y que fueron adaptados para las otras medidas de $I S$ (figuras 23-24). Las figuras muestran la dispersión de la distorsión máxima de entrepiso para cada parámetro considerado en este estudio y para el marco de 18 niveles ante movimientos de suelo MB característico de la ciudad de México. Para los casos de $I_{N p}$ e $I_{B}$ estos fueron calculados con los valores de $\alpha$ y $\alpha_{1}, \alpha_{2}$ y $\alpha_{3}$ descritos anteriormente. Se observa que la 
dispersión de la demanda sísmica es mucho menor cuando se utiliza $I_{B}$ en comparación con los otros parámetros analizados, lo cual indica las ventajas de esta medida de intensidad sísmica con respecto a las otras. Se hace notar que la reducción de la incertidumbre en la respuesta sísmica es de gran utilidad para establecer criterios de selección de acelerogramas para análisis y diseño sísmico, así como para estudios de vulnerabilidad y confiabilidad estructural.

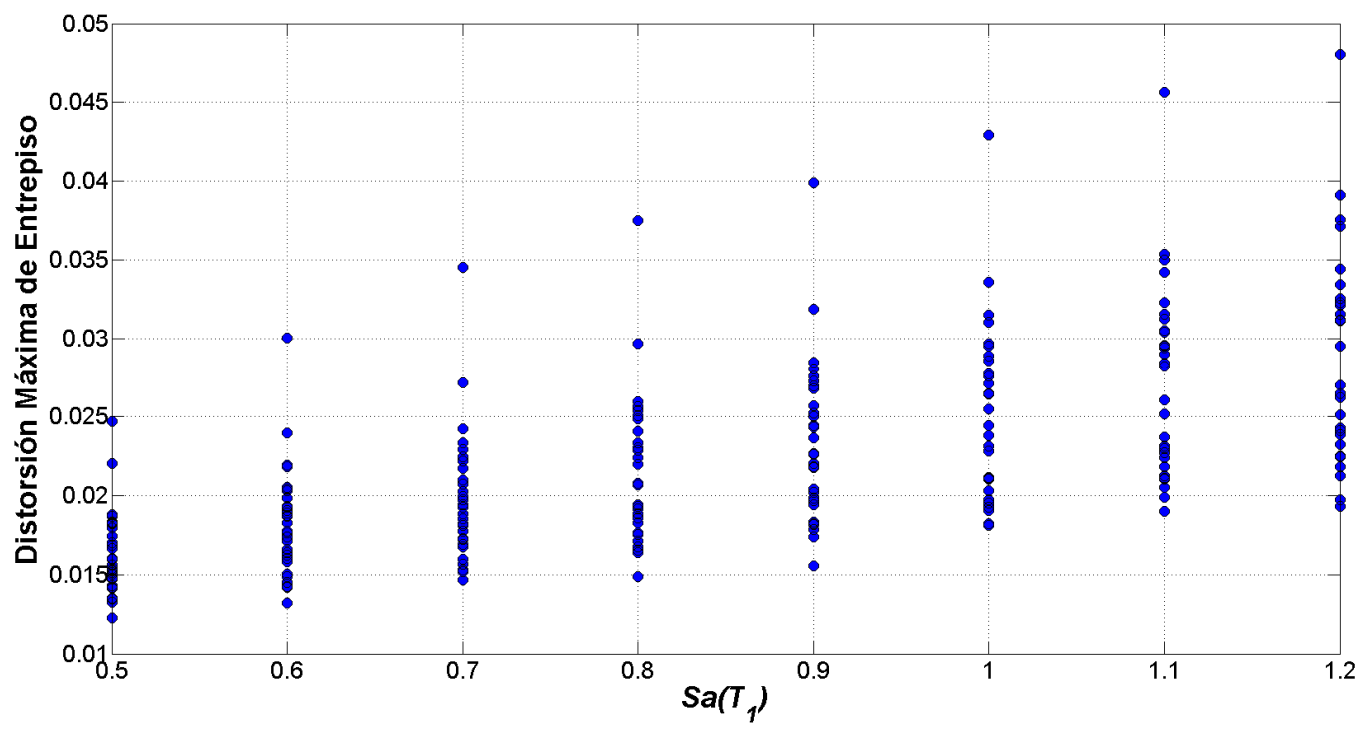

Figura 22. Análisis dinámico incremental del marco de 18 niveles sometido a los registros sísmicos obtenidos de suelo MB usando $S a\left(T_{1}\right)$

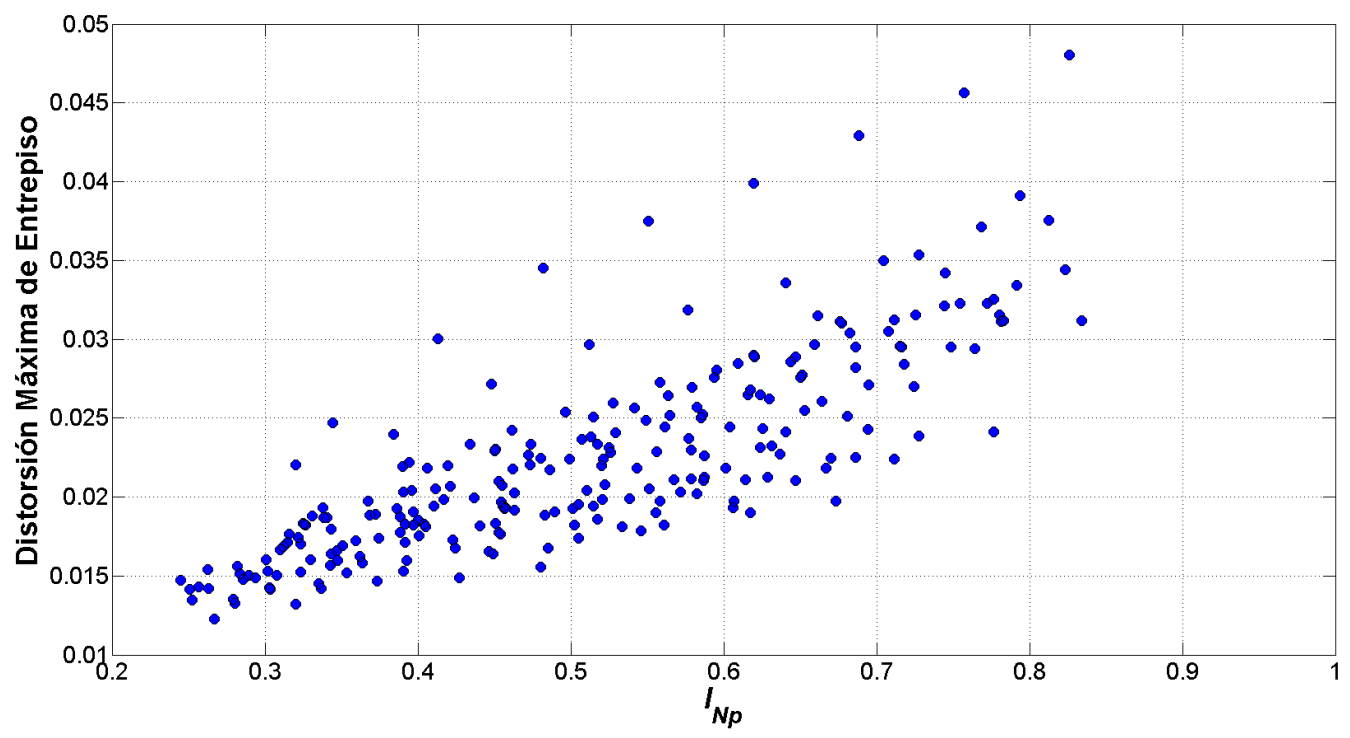

Figura 23. Análisis dinámico incremental del marco de 18 niveles sometido a los registros sísmicos obtenidos de suelo MB usando $I_{N p}$ 


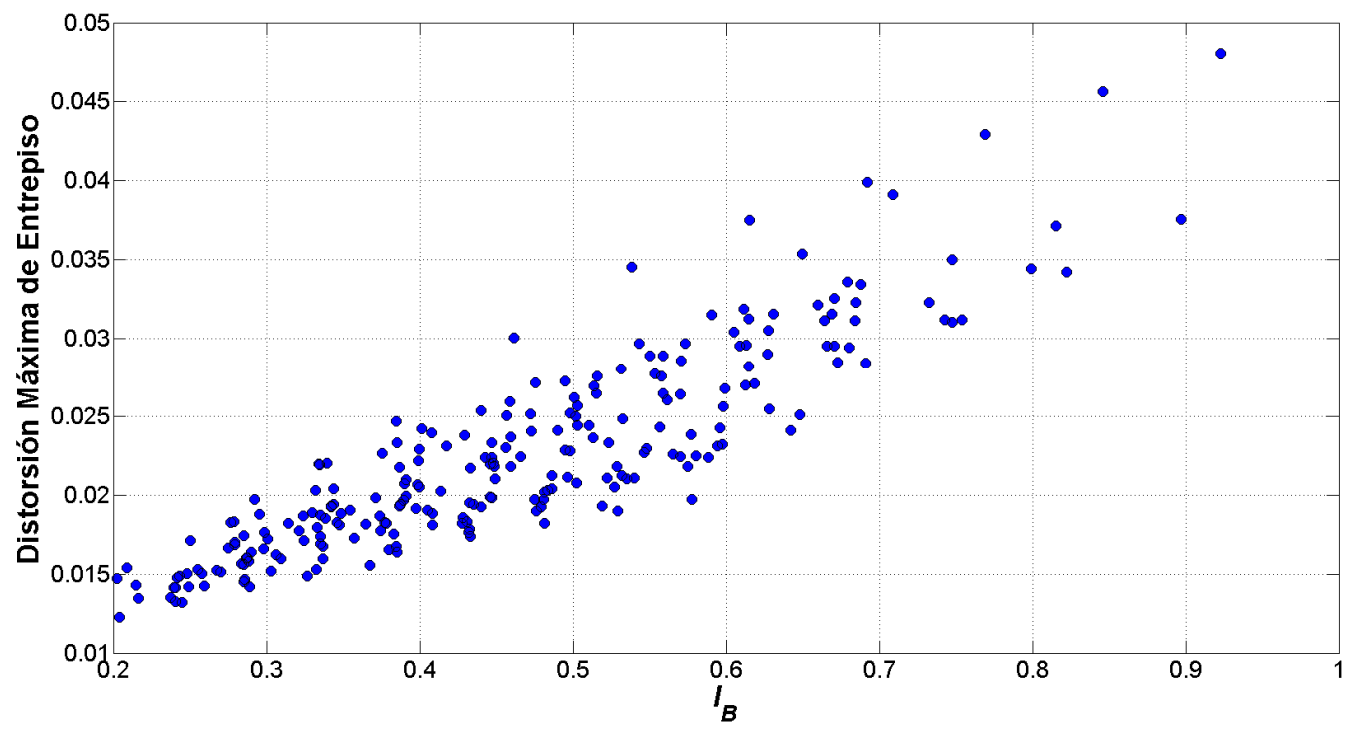

Figura 24. Análisis dinámico incremental del marco de 18 niveles sometido a los registros sísmicos obtenidos de suelo MB usando $I_{B(S a, S a, S a)}$

\section{COMPARACIÓN DE $I_{B(S a, S a, S a)}$ CONTRA $S a\left(T_{1}\right)$}

Con la finalidad de demostrar que los exponentes $\alpha_{1}, \alpha_{2}$ y $\alpha_{3}$ obtenidos en este estudio, para los cuales la medida $I_{B}$ presenta menores desviaciones estándar, no dependen de los registros previamente utilizados en este trabajo. Se seleccionaron 40 registros de manera aleatoria tomados de la base de datos del Centro de Investigación de Ingeniería Sísmica del Pacifico (Base de Datos NGA), con estos registros se obtuvieron las desviaciones estándar para el caso de $I_{B(S a, S a, S a)}$ tomando en cuenta únicamente los exponentes $\alpha_{1}, \alpha_{2}$ y $\alpha_{3}$ iguales a $1,0.4$ y 0.2 respectivamente, para los 6 marcos estructurales en estudio. La figura 25 permite realizar una comparación justa entre $I_{B(S a, S a, S a)}$ y $S a\left(T_{1}\right)$, debido a que los registros aleatorios utilizados para obtener las desviaciones estándar de $I_{B(S a, S a, S a)}$ no fueron utilizados previamente para definir $\alpha_{1}, \alpha_{2}$ y $\alpha_{3}$. En la figura 25 se observa que para la mayoría de los marcos estructurales $I_{B(S a, S a, S a)}$ presenta menores desviaciones estándar que $S a\left(T_{1}\right)$; sin embargo, para el marco de 4 niveles la desviación estándar es bastante similar para ambas medidas de intensidad sísmica esto debido a la baja altura del marco sobre el cual no se presenta un efecto considerable del segundo modo de vibración. 


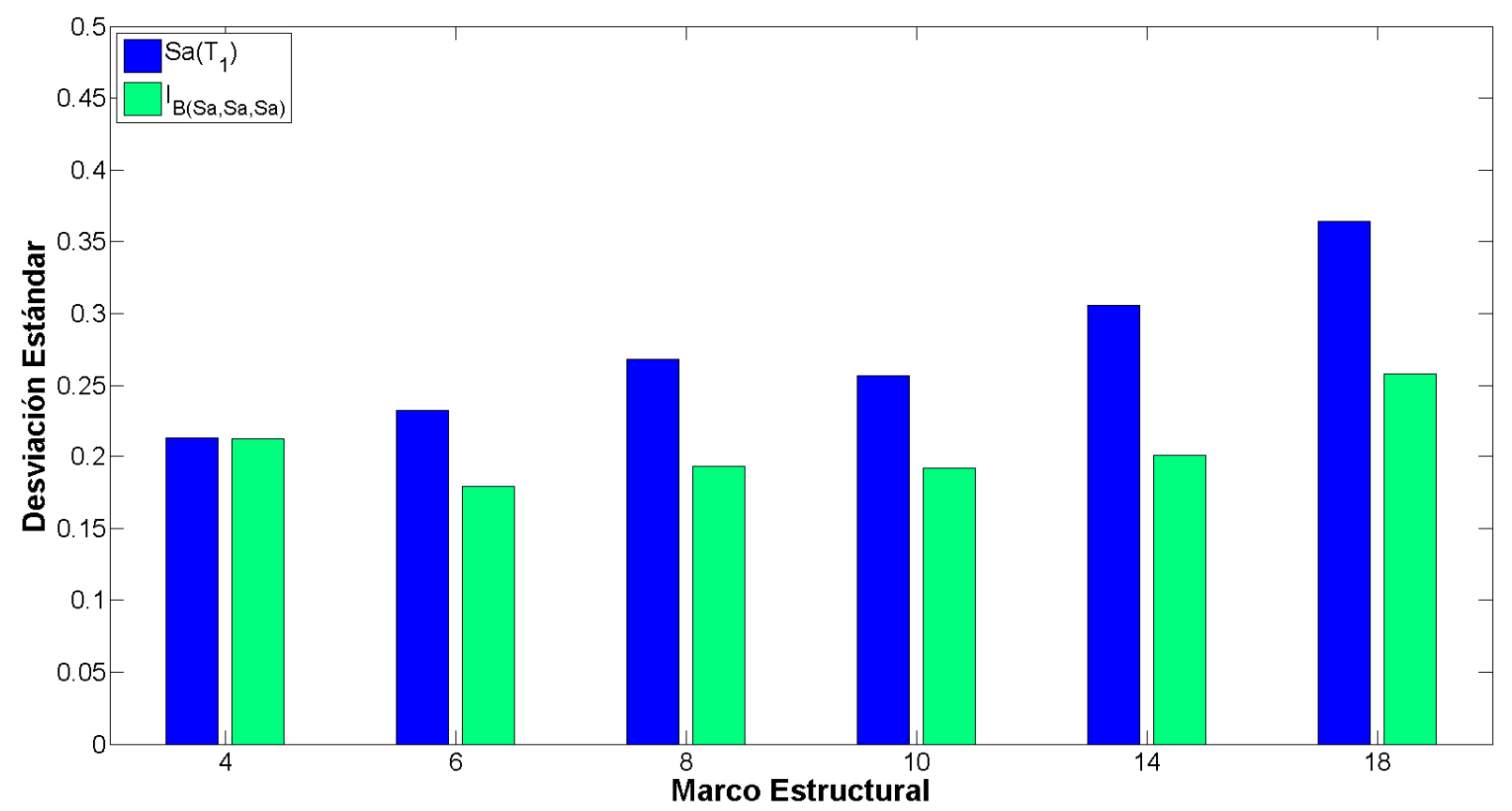

Figura 25. Comparación de $\sigma_{\ln (\gamma)}$ para los marco de 4, 6, 8, 10, 14 y 18 niveles y para los 40 registros de tipos aleatorio

\section{CONCLUSIONES}

Se realizó un análisis sobre la eficiencia de algunas medidas de intensidad sísmica representativas de la forma espectral, por ser esta característica de gran importancia como predictor de la respuesta estructural de edificaciones. Para ello, varias estructuras de acero se sometieron a movimientos sísmicos obtenidos en distintos tipos de suelo, que van desde muy rígidos como roca sana hasta muy blandos como los que se encuentran en la zona blanda del valle de México. Los resultados obtenidos muestran que los parámetros $S a\left(T_{1}\right)$ e $I_{N p}$ reflejaron ser buenos predictores de la respuesta estructural en términos de la distorsión máxima de entrepiso; sin embargo, la medida $I_{B}$, que incorpora información de los modos superiores y toma en cuenta el comportamiento no lineal, resultó ser el parámetro más eficiente en la mayoría de los tipos de suelo.

De acuerdo con los resultados obtenidos en este estudio se puede concluir que $S a\left(T_{1}\right)$ es buen predictor de la respuesta estructural siempre y cuando la estructura en estudio se encuentre en un intervalo de comportamiento lineal y dominado por su periodo fundamental de vibración. Por otro lado, $I_{N p}$ resultó ser un buen predictor de la respuesta sísmica cuando la estructura exhibe presencia de comportamiento no lineal y es dominada por su periodo fundamental de vibración. La conclusión más importante del presente estudio es que $I_{B}$ es buen predictor de la respuesta sísmica cuando la estructura exhibe presencia de comportamiento no lineal y los modos superiores de vibración tienen una participación significativa, lo cual es válido para edificaciones desplantadas en distinto tipo de suelo. En otras palabras, la alta eficiencia de $I_{B}$ no depende del tipo de suelo en el cual se registran los movimientos sísmicos, lo que indica el gran potencial de la medida de intensidad sísmica analizada.

Los resultados obtenidos de manera preliminar sugieren que es aceptable utilizar $\alpha=0.4$ para $I_{N p}$, dicho valor coincide con el obtenido previamente por Bojórquez y Iervolino (2011). Por otro lado, se 
sugiere que para calcular la medida de intensidad $I_{B}$ se utilicen valores de $\alpha_{1}=1, \alpha_{2}=0.4$ y $\alpha_{3}=0.2$ para los tres casos sobresalientes, de acuerdo con los resultados mostrados.

Al analizar los tres casos de $I_{B}$ se puede concluir que $I_{B(S a, S a, S a)}$ y $I_{B(S a, V, S a)}$ presentan una desviación estándar similar para la mayoría de los tipos de suelo y estructuras, mientras que para $I_{B(V, V, S a)}$ la desviación estándar tiende a disminuir para algunos tipos de suelos a medida que se incrementa la altura de la estructura con respecto a $I_{B(S a, S a, S a)}$ y $I_{B(S a, V, S a)}$. Como muestran los resultados, los tres casos de $I_{B}$ presentan una eficiencia similar por tal motivo se recomienda utilizar $I_{B(S a, S a, S a)}$ como medida de intensidad sísmica, ya que resulta más fácil su empleo en comparación con los otros dos casos ya que únicamente se necesita el espectro de pseudo-aceleración para estimarlo.

El estudio permitió verificar la importancia de utilizar $I_{B}$ como medida de intensidad sísmica ya que es capaz de predecir con buena aproximación el comportamiento sísmico de estructuras. Aunque es necesario realizar mayores estudios de eficiencia, se puede concluir que tanto $I_{N p}$ como $I_{B}$, son buenos candidatos para reflejar el potencial destructivo de un movimiento sísmico, y podrían representar la pieza clave para el análisis de peligro y riesgo sísmico.

Finalmente, es importante mencionar que los resultados obtenidos en el presente trabajo dependen fuertemente de las edificaciones seleccionadas, los criterios para su modelación, así como los registros sísmicos empleados; sin embargo, como se menciono en varias partes del trabajo, algunos autores han utilizado otro tipo de estructuración y acelerogramas y han hecho ver que el parámetro $N_{p}$ en el cual se basa la nueva propuesta $I_{B}$ es bastante prometedor como parte de una medida de intensidad sísmica.

\section{AGRADECIMIENTOS}

Se agradecen los valiosos comentarios de los revisores anónimos los cuales sirvieron para mejorar la calidad del estudio presentado. Este trabajo se desarrolló con el apoyo económico brindado por El Consejo Nacional de Ciencia y Tecnología a través del Proyecto CB-2011-01-167419 y la Beca otorgada al segundo autor. Se agradece el apoyo de la Universidad Autónoma de Sinaloa dentro del proyecto PROFAPI 2013/025 así como de la Universidad Nacional Autónoma de México mediante DGAPAPAPIIT IN107011.

\section{REFERENCIAS}

Alcántara, L, E Ovando y M A Macías (2009), "Estimación de espectros de respuesta en la ciudad de puebla utilizando redes neuronales artificiales," XVI Congreso Nacional de Ingeniería Sísmica, Puebla, México.

Aptikaev, F F (1982), "On the correlations of MM intensity with parameters of ground shaking", 7th European Conference on Earthquake Engineering, Atenas, Grecia.

Araya, R y G R Saragoni (1980), "Capacidad de los movimientos sísmicos de producir daño estructural”, Reporte SES I 7/80, División de Ingeniería Estructural, Departamento de Ingeniería, Universidad de Chile, Santiago, Chile.

Arias, A (1970), “A measure of earthquake intensity", in Seismic Design for Nuclear Power Plants, editado por R. J. Hansen, Massachusetts Institute of Technology Press, pp. 438-483. 
Baker, J W y C A Cornell (2004), "Choice of a vector ground motion intensity measures for Seismic Demand Hazard Analysis", 13th World Conference on Earthquake Engineering. Agosto 1-6, Vancouver, Canada.

Baker, J W y C A Cornell. (2005), "A vector-valued ground motion intensity measure consisting of spectral acceleration and epsilon", Earthquake Engineering \& Structural Dynamics, vol. 34, pp. 1193 - 1217.

Baker, J W y C A Cornell (2006), "Spectral shape, epsilon and record selection”, Earthquake Engineering \& Structural Dynamics, vol. 35, pp. 1077-1095.

Bazzurro, P y C A Cornell (2002), "Vector-valued probabilistic seismic hazard analysis (VPSHA)", Proceedings of the Seventh U.S. National Conference on Earthquake Engineering, Boston, MA.

Bojórquez, E, I Iervolino y G Manfredi (2008), "Evaluating a new proxy for spectral shape to be used as an intensity measure", Seismic Engineering Conference, AIP Conference Proceedings, vol. 1020, pp. 1599-1606.

Bojórquez, E y I Iervolino (2011), "Spectral shape proxies and nonlinear structural response", Soil Dynamics and Earthquake Engineering, vol. 31, pp 996-1008.

Bojórquez, E, I Iervolino y A Reyes-Salazar (2011). "Which spectral shape really matter to predict nonlinear structural response: application to steel frames", Paper 05-175, 8th International Conference on Urban Earthquake Engineering (CUEE), Tokio Japan.

Bojórquez, E, I Iervolino, A Reyes-Salazar y S E Ruiz (2012a), "Comparing vector-valued intensity measures for fragility analysis of steel frames in the case of narrow-band ground motions", Engineering Structures, vol. 45, pp. 472-480.

Bojórquez, E, J Bojórquez, S E Ruiz y A Reyes-Salazar (2012b). "Prediction of inelastic response spectra using artificial neural networks". Mathematical Problems in Engineering, vol. 2012.

Bojórquez, E (2014), "A new generalized ground motion intensity measure part I: mathematical formulation", en preparación.

Bojórquez, E, R Chavez, S E Ruiz y A Reyes-Salazar (2014)," A new generalized ground motion intensity measure part II: application", en preparación.

Buratti, N (2011), "Confronto tra le performance di diverse misure di intensità dello scuotimento sísmico", Congreso Nacional de Ingeniería de Italia, ANIDIS, Bari Italia.

Buratti, N (2012), "A comparison of the performances of various ground-motion intensity measures", The 15th World Conference on Earthquake Engineering, Lisbon, Portugal, 24-28 September.

Carr, A (2008), "RUAUMOKO inelastic dynamic analysis program", Departament of Civil Engineering, University of Cantenbury, Nueva Zelanda.

Cordova, P P, G G Dierlein, S S F Mehanny y C A Cornell (2001), "Development of a two parameter seismic intensity measure and probabilistic assessment procedure", The second U.S.-Japan Workshop on Performance-Based Earthquake Engineering Methodology for Reinforce Concrete Building Structures, Sapporo, Hokkaido 2001, pp. 187-206.

FEMA (1994), "NEHRP Recommended provisions for the development of seismic regulations for new buildings".

Housner, G W (1952), "Spectrum intensities of strong motion earthquakes", Proceedings, Symposium on Earthquake and Blast Effects on Structures, Earthquake Engineering Research Institute. 
Housner, G W (1970), "Strong ground motion", In Earthquake Engineering, editado por R. L. Wiegel, Prentice-Hall Inc.

Housner, G W (1975), "Measures of severity of ground shaking", U.S. Conference on Earthquake Engineering. Earthquake Engineering Research Institute.

Housner, G W y P C Jennings (1964), "Generation of artificial earthquakes”, Journal of the Engineering Mechanics Division, vol. 90, Proceedings paper 3806.

Iervolino, I y C A Cornell (2005), "Records selection for nonlinear seismic analysis of structures", Earthquake Spectra, vol. 21, pp. 685-713.

Kurama, Y C y K T Farrow (2003), "Ground motion scaling methods for different site conditions and structure characteristics", Earthquake Engineering and Structural Dynamics, vol. 32, pp. 24252450 .

Luco, N (2002), "Probabilistic seismic demand analysis, SMRF connection fractures, and near-source effects", Ph.D. Thesis, Stanford University.

Mehanny, S S F (2009), "A broad-range power-law form scalar-based seismic intensity measure", Engineering Structures, vol. 31, pp. 1354-1368.

Mehanny, S S F y G G Deierlein (2000), "Modelling of assessment of seismic performance of composite frames with reinforced concrete columns and steel beams". Report 135, Department of Civil and Enviromental Engineering, Stanford University, Sanford.

Modica, A y P Stafford (2014), "Vector fragility surfaces for reinforced concrete frames in Europe", Bulletin of Earthquake Engineering, 2014.

Park, Y J, A H S Ang y Y K Wen (1985), "Seismic damage analysis of reinforced concrete buildings", Journal of Structural Engineering, vol. 111, pp. 740-757.

RCDF (2004), "Reglamento de Construcciones del Distrito Federal".

Riddell, R y J E Garcia (2001), "Hysteretic energy spectrum and damage control", Earthquake Engineering \& Structural Dynamics, vol. 30, pp. 1791-1816.

Sarma, S K y K S Yang (1987), "An evaluation of strong motion records and a new parameter A95", Earthquake Engineering and Structural Dynamics, vol. 15, pp. 119-132.

Shome, N, C A Cornell, P Bazzurro y J E Carballo (1998), "Earthquakes, records, and nonlinear responses", Earthquake Spectra, vol. 14(3), pp. 469 - 500.

Shome, N (1999), "Probabilistic seismic demand analysis of nonlinear structures", Ph.D. Thesis, dissertation, Department of Civil and Environmental Engineering, Stanford University.

Terán-Gilmore, A y O Jirsa (2007), "Energy demands for seismic design against low cycle fatigue", Earthquake Engineering and Structural Dynamics, vol. 36, pp. 383-404.

Tothong, P y N Luco (2007), "Probabilistic seismic demand analysis using advanced ground motion intensity measures", Earthquake Engineering and Structural Dynamics, vol. 36, pp. 1837-1860.

Vamvatsikos, D y C A Cornell (2002), "Incremental dynamic analysis", Earthquake Engineering and Structural Dynamics, vol. 31, pp. 491-514.

Von-Thun, J L, L H Rochin, G A Scott y J A Wilson (1988), "Earthquake ground motions for design and analysis of dams, in: Earthquake Engineering and Soil Dynamics II - Recent Advance in GroundMotion Evaluation”, Geotechnical Special Publication 20 ASCE, New York, pp. 463-481. 